\title{
The Density Theorem and the Homogeneous Approximation Property for Gabor Frames
}

\author{
Christopher Heil \\ School of Mathematics, Georgia Institute of Technology, Atlanta, GA 30332 USA \\ heil@math.gatech.edu
}

Summary. The Density Theorem for Gabor Frames is a fundamental result in timefrequency analysis. Beginning with Baggett's proof that a rectangular lattice Gabor system $\left\{e^{2 \pi i \beta n t} g(t-\alpha k)\right\}_{n, k \in \mathbf{Z}}$ must be incomplete in $L^{2}(\mathbf{R})$ whenever $\alpha \beta>1$, the necessary conditions for a Gabor system to be complete, a frame, a Riesz basis, or a Riesz sequence have been extended to arbitrary lattices and beyond. The first partial proofs of the Density Theorem for irregular Gabor frames were given by Landau in 1993 and by Ramanathan and Steger in 1995. A key fact proved by Ramanathan and Steger is that irregular Gabor frames possess a certain Homogeneous Approximation Property (HAP), and that the Density Theorem is a consequence of this HAP. This chapter provides a brief history of the Density Theorem and a detailed account of the proofs of Ramanathan and Steger. Furthermore, we show that the techniques of Ramanathan and Steger can be used to give a full proof of a general version of Density Theorem for irregular Gabor frames in higher dimensions and with finitely many generators.

\section{Introduction}

\subsection{Frames and Gabor Systems}

Frames provide basis-like but generally nonunique representations of vectors in a Hilbert space. Moreover, these expansions possess a variety of desirable stability properties. One of these is inherent in the definition itself: A sequence $\left\{f_{n}\right\}_{n \in \mathbf{N}}$ is a frame if the $\ell^{2}$-norm of the frame coefficients $\left\{\left\langle f, f_{n}\right\rangle\right\}_{n \in \mathbf{N}}$ of $f \in$ $H$ is an equivalent norm for $H$. Thus a perturbation of $f$ is directly reflected in the size of the frame coefficients, and vice versa. Moreover, it follows from this equivalence of norms that there exist expansions of the form $f=\sum c_{n}(f) f_{n}$ for each $f \in H$. The coefficients $c_{n}(f)$ are not unique in general, but there is a canonical choice, and for this choice the expansions converge unconditionally (regardless of ordering). A frame for which the coefficients are unique for each $f$ is called a Riesz basis, and is the image of an orthonormal basis under a 
continuous bijection of $H$ onto itself. Precise definitions of all terminology are given in Section 2 below.

Frames were first introduced by Duffin and Schaeffer [19] in the context of nonharmonic Fourier expansions, and today they have applications in a wide range of areas. We will be especially interested in a particular class of frames for $L^{2}\left(\mathbf{R}^{d}\right)$ whose elements are simply generated from a single generator in $L^{2}\left(\mathbf{R}^{d}\right)$. Given $g \in L^{2}\left(\mathbf{R}^{d}\right)$, called a window function or an atom, and given a sequence $\Lambda$ of points in the time-frequency plane $\mathbf{R}^{d} \times \mathbf{R}^{d}=\mathbf{R}^{2 d}$, the Gabor system generated by $g$ and $\Lambda$ is

$$
\mathcal{G}(g, \Lambda)=\left\{e^{2 \pi i \xi \cdot t} g(t-x)\right\}_{(x, \xi) \in \Lambda}=\left\{M_{\xi} T_{x} g\right\}_{(x, \xi) \in \Lambda},
$$

where $T_{x} g(t)=g(t-x)$ is translation and $M_{\xi} g(t)=e^{2 \pi i t \cdot \xi} g(t)$ is modulation. That is, the Gabor system is generated by applying a discrete collection of time-frequency shift operators $M_{\xi} T_{x}$ to the window $g$. The Gabor system is a frame if there exist constants $A, B>0$ such that

$$
\forall f \in L^{2}\left(\mathbf{R}^{d}\right), \quad A\|f\|_{2}^{2} \leq \sum_{(x, \xi) \in \Lambda}\left|\left\langle f, M_{\xi} T_{x} g\right\rangle\right|^{2} \leq B\|f\|_{2}^{2} .
$$

We will be concerned in this chapter with the question of when a Gabor system can be complete, a frame, a Riesz basis, or a Riesz sequence (a Riesz basis for its closed span) in $H$. There is a rich literature on this subject, which we will only attempt to briefly review, while the main body of the chapter will be concerned with an approach to this question introduced by Ramanathan and Steger in [47]. For a detailed survey of the history, context, and evolution of the Density Theorem, we refer to [30].

\subsection{Gaussian Windows}

We begin our history with the special case of the Gaussian window $\varphi(t)=$ $2^{1 / 4} e^{-\pi t^{2}}$ in one dimension. Von Neumann [43, p. 406] claimed without proof that if $\Lambda$ is the unit lattice $\Lambda=\mathbf{Z}^{2}$, then the Gabor system $\mathcal{G}\left(g, \mathbf{Z}^{2}\right)=$ $\left\{M_{n} T_{k} \varphi\right\}_{k, n \in \mathbf{Z}}$ is complete in $L^{2}(\mathbf{R})$. This claim was proved to be correct in [6], [46], and [1]. However, completeness is quite a weak property. It only means that the finite linear span is dense, so every vector $f \in L^{2}(\mathbf{R})$ can be approximated as well as we like by some finite linear combination of the elements of $\mathcal{G}\left(\varphi, \mathbf{Z}^{2}\right)$, but it gives us no information about what those linear combinations are.

Gabor [23] conjectured more: he wrote that each $f \in L^{2}(\mathbf{R})$ can actually be represented in the form

$$
f=\sum_{k, n \in \mathbf{Z}} c_{k n}(f) M_{n} T_{k} \varphi
$$

for some scalars $c_{k n}(f)$. This is not a consequence of completeness. Gabor's claim was proved by Janssen [34] to be correct-but only in a weak sense, 
as he proved that the series in (1) converge only in the sense of tempered distributions and not in the norm of $L^{2} \cdot \mathcal{G}\left(\varphi, \mathbf{Z}^{2}\right)$ is neither a frame nor a Riesz basis for $L^{2}(\mathbf{R})$.

\subsection{The Density Theorem for Rectangular Lattice Gabor Systems}

As we have seen, the Gabor system generated by the Gaussian window with respect to the unit lattice is not particularly useful. However, we can try other windows and other index sets, such as a rectangular lattice of the form $\Lambda=$ $\alpha \mathbf{Z} \times \beta \mathbf{Z}$. Indeed, Daubechies, Grossmann, and Morlet sparked a revitalization of interest in frame theory when they proved in [16] that for each $\alpha, \beta>0$ with $\alpha \beta \leq 1$ it is possible to construct a compactly supported $g$ such that $\mathcal{G}(g, \alpha \mathbf{Z} \times \beta \mathbf{Z})$ forms a frame for $L^{2}(\mathbf{R})$. When $\alpha \beta<1$, we can even do this with $g \in C_{c}^{\infty}(\mathbf{R})$. For $\alpha \beta=1$, if we let $g$ be the characteristic function of the interval $[0, \alpha]$, then $\mathcal{G}\left(g, \alpha \mathbf{Z} \times \frac{1}{\alpha} \mathbf{Z}\right)$ is an orthonormal basis for $L^{2}(\mathbf{R})$. Thus, at least when $\alpha \beta<1$, we can form Gabor frames using windows which are very well-localized in the time-frequency plane.

Two later fundamental results for Gabor systems on rectangular lattices show that the properties of the "Painless Nonorthogonal Expansions" of [16] are actually typical of Gabor systems. The first is the following Density Theo$r e m$, which states that the value of $\alpha \beta$ distinguishes between the cases where $\mathcal{G}(g, \alpha \mathbf{Z} \times \beta \mathbf{Z})$ can be complete, a frame, a Riesz basis, or a Riesz sequence. Only the value of the product is relevant since by applying the unitary dilation operator $D_{r} g(t)=r^{1 / 2} g(r t), \mathcal{G}(g, \alpha \mathbf{Z} \times \beta \mathbf{Z})$ is complete, a frame, a Riesz basis, or a Riesz sequence if and only if the same is true of $\mathcal{G}\left(D_{r} g, \frac{\alpha}{r} \mathbf{Z} \times r \beta \mathbf{Z}\right)$.

Theorem 1 (Density Theorem for Rectangular Lattices). Let $g \in$ $L^{2}(\mathbf{R})$ and let $\Lambda=\alpha \mathbf{Z} \times \beta \mathbf{Z}$ where $\alpha, \beta>0$. Then the following statements hold.

(a) If $\alpha \beta>1$, then $\mathcal{G}(g, \alpha \mathbf{Z} \times \beta \mathbf{Z})$ is incomplete in $L^{2}(\mathbf{R})$.

(b) If $\mathcal{G}(g, \alpha \mathbf{Z} \times \beta \mathbf{Z})$ is a frame for $L^{2}(\mathbf{R})$, then $0<\alpha \beta \leq 1$.

(c) $\mathcal{G}(g, \alpha \mathbf{Z} \times \beta \mathbf{Z})$ is a Riesz basis for $L^{2}(\mathbf{R})$ if and only if it is a frame for $L^{2}(\mathbf{R})$ and $\alpha \beta=1$.

(d) If $\mathcal{G}(g, \alpha \mathbf{Z} \times \beta \mathbf{Z})$ is a Riesz sequence in $L^{2}(\mathbf{R})$, then $\alpha \beta \geq 1$.

The Density Theorem and its extensions will be the focus of this chapter, but we also mention here a second result which explains why there is such a difference in quality between the constructions of [16] for the cases $\alpha \beta<1$ and $\alpha \beta=1$. This is the Balian-Low Theorem, which states that the generator of any rectangular lattice Gabor Riesz basis must be poorly localized in the time-frequency plane.

Theorem 2 (Classical Balian-Low Theorem). If $g \in L^{2}(\mathbf{R})$ is such that $\mathcal{G}(g, \alpha \mathbf{Z} \times \beta \mathbf{Z})$ is a Riesz basis for $L^{2}(\mathbf{R})$, then 


$$
\left(\int_{\mathbf{R}}|t g(t)|^{2} d t\right)\left(\int_{\mathbf{R}}|\xi \hat{g}(\xi)|^{2} d \xi\right)=\infty .
$$

As a consequence, it is "redundant" (non-Riesz basis) Gabor frames that are usually used in practice.

\subsection{Brief History of the Density Theorem}

Rectangular lattice Gabor systems are especially attractive because there are so many tools available for studying them. We will mention some of these while giving a brief review of the history of the Density Theorem.

Part (a) of Theorem 1 was proved by Baggett in [2]. The time-frequency operators $M_{\beta n} T_{\alpha k}$ corresponding to the rectangular lattice $\alpha \mathbf{Z} \times \beta \mathbf{Z}$ generate a von Neumann algebra, and Baggett made use of the representation theory of the discrete Heisenberg group to derive his proof. For an exposition of Baggett's operator-theoretic proof, see [21].

Part (a) was also proved, for the special case that the product $\alpha \beta$ is rational by Daubechies [13]. Her proof relied on the Zak transform, which is another "algebraic" tool in the sense that it is dependent on the fact that the index set is a rectangular lattice. This proof is constructive in the sense that if $g$ and a rational $\alpha \beta>1$ are given, then it constructs a nonzero function that is orthogonal to all the elements of $\mathcal{G}(g, \alpha \mathbf{Z} \times \beta \mathbf{Z})$.

Daubechies also noted in [13] that a proof for general $\alpha \beta$ can be inferred from results of Rieffel [48] on coupling constants of $C^{*}$-algebras (however, no part of the Density Theorem is explicitly stated in [48]). Another proof of part (a), also based on coupling constants, was given by Daubechies, H. Landau, and Z. Landau in [17], and other proofs can be found in [22] and [9].

Speaking of the operator-theoretic approaches by Baggett and Rieffel, Gröchenig [25, p. 139] remarks:

The power of this abstract mathematical theory seems to have been slightly suspicious to applied mathematicians ...

and as a consequence they inspired a wide range of new and different approaches to issues connected with the Density Theorem. In particular, while part (b) is a special case of part (a), several different and revealing proofs of part (b) have been found (i.e., proofs that show that $\mathcal{G}(g, \alpha \mathbf{Z} \times \beta \mathbf{Z})$ cannot be a frame when $\alpha \beta>1$, but which do not show that $\mathcal{G}(g, \alpha \mathbf{Z} \times \beta \mathbf{Z})$ must be incomplete). For example, Janssen [35] gave a "simple" proof of parts (b) and (c) based on the algebraic structure of the rectangular lattice $\alpha \mathbf{Z} \times \beta \mathbf{Z}$ and the remarkable Wexler-Raz biorthogonality relations for Gabor frames $\mathcal{G}(g, \alpha \mathbf{Z} \times \beta \mathbf{Z})$.

Before stating the Wexler-Raz relations, let us recall a few basic facts. If $\mathcal{G}(g, \alpha \mathbf{Z} \times \beta \mathbf{Z})$ is a frame, then the frame operator

$$
S f=\sum_{k, n \in \mathbf{Z}}\left\langle f, M_{\beta n} T_{\alpha k} g\right\rangle M_{\beta n} T_{\alpha k} g
$$


is a positive, invertible mapping of $L^{2}(\mathbf{R})$ onto itself. The canonical dual frame of $\mathcal{G}(g, \alpha \mathbf{Z} \times \beta \mathbf{Z})$ is $S^{-1}(\mathcal{G}(g, \alpha \mathbf{Z} \times \beta \mathbf{Z}))$. However, it is easy to see that $S$ commutes with $M_{\beta n} T_{\alpha k}$ for $k, n \in \mathbf{Z}$, hence $S^{-1}$ commutes with them as well, and so this dual frame actually is the Gabor frame $\mathcal{G}(\tilde{g}, \alpha \mathbf{Z} \times \beta \mathbf{Z})$ where $\tilde{g}=S^{-1} g$. By expanding the equalities $f=S S^{-1} f=S^{-1} S f$, we obtain the frame expansions

$$
f=\sum_{k, n \in \mathbf{Z}}\left\langle f, M_{\beta n} T_{\alpha k} \tilde{g}\right\rangle M_{\beta n} T_{\alpha k} g=\sum_{k, n \in \mathbf{Z}}\left\langle f, M_{\beta n} T_{\alpha k} g\right\rangle M_{\beta n} T_{\alpha k} \tilde{g},
$$

for $f \in L^{2}(\mathbf{R})$, where these series converge unconditionally in the norm of $L^{2}(\mathbf{R})$. In general, however, the coefficients in these expansions need not be unique. It is important to note that the structure of the index set $\Lambda=\alpha \mathbf{Z} \times \beta \mathbf{Z}$ is critical to these remarks - for an arbitrary sequence $\Lambda$ the dual frame of $\mathcal{G}(g, \Lambda)$ need not be another Gabor frame, cf. [5].

The next result is actually just a special case of the Wexler-Raz relations, cf. [25, Thm. 7.3.1], and see [30] for more detailed references.

Theorem 3 (Wexler-Raz Biorthogonality Relations). Let $g \in L^{2}(\mathbf{R})$ and $\alpha, \beta>0$ be such that $\mathcal{G}(g, \alpha \mathbf{Z} \times \beta \mathbf{Z})$ is a frame for $L^{2}(\mathbf{R})$, with canonical dual frame $\mathcal{G}(\tilde{g}, \alpha \mathbf{Z} \times \beta \mathbf{Z})$. Then $\mathcal{G}\left(g, \frac{1}{\beta} \mathbf{Z} \times \frac{1}{\alpha} \mathbf{Z}\right)$ and $\mathcal{G}\left(\tilde{g}, \frac{1}{\beta} \mathbf{Z} \times \frac{1}{\alpha} \mathbf{Z}\right)$ are biorthogonal, specifically,

$$
\frac{1}{\alpha \beta}\left\langle M_{\frac{n}{\alpha}} T_{\frac{k}{\beta}} g, M_{\frac{n^{\prime}}{\alpha}} T_{\frac{k^{\prime}}{\beta}} \tilde{g}\right\rangle=\delta_{k k^{\prime}} \delta_{n n^{\prime}} .
$$

Note that a frame has a biorthogonal system if and only if it is a Riesz basis. However, in the Wexler-Raz relations it is not the frame $\mathcal{G}(g, \alpha \mathbf{Z} \times \beta \mathbf{Z})$ which has a biorthogonal system, but rather the Gabor system $\mathcal{G}\left(g, \frac{1}{\beta} \mathbf{Z} \times \frac{1}{\alpha} \mathbf{Z}\right)$ defined on the adjoint lattice $\frac{1}{\beta} \mathbf{Z} \times \frac{1}{\alpha} \mathbf{Z}$.

Part (b) of the Density Theorem follows directly from the Wexler-Raz relations. Using the notation given above, if $\mathcal{G}(g, \alpha \mathbf{Z} \times \beta \mathbf{Z})$ is a frame then from equation (3) we have that $\alpha \beta=\langle g, \tilde{g}\rangle$. However, we know that $S^{-1}$ is a positive operator that commutes with $M_{\beta n} T_{\alpha k}$, so by applying the frame expansions in (2) to the function $f=g$ (and recalling that $\tilde{g}=S^{-1} g$ ), we obtain the following:

$$
\begin{aligned}
\alpha \beta=\langle g, \tilde{g}\rangle & =\left\langle\sum_{k, n \in \mathbf{Z}}\left\langle g, M_{\beta n} T_{\alpha k} S^{-1} g\right\rangle M_{\beta n} T_{\alpha k} g, S^{-1} g\right\rangle \\
& =\sum_{k, n \in \mathbf{Z}}\left\langle g, M_{\beta n} T_{\alpha k} S^{-1} g\right\rangle\left\langle S^{-1} M_{\beta n} T_{\alpha k} g, g\right\rangle \\
& =\sum_{k, n \in \mathbf{Z}}\left|\left\langle g, M_{\beta n} T_{\alpha k} S^{-1} g\right\rangle\right|^{2}
\end{aligned}
$$




$$
\begin{aligned}
& \geq\left|\left\langle g, S^{-1} g\right\rangle\right|^{2} \\
& =|\langle g, \tilde{g}\rangle|^{2}=(\alpha \beta)^{2} .
\end{aligned}
$$

Consequently, $\alpha \beta \leq 1$, which proves part (b) of Theorem 1 .

Further, if $\mathcal{G}(g, \alpha \mathbf{Z} \times \beta \mathbf{Z})$ is a Riesz basis then it follows from basic frame principles that it is biorthogonal to its canonical dual frame $\mathcal{G}(\tilde{g}, \alpha \mathbf{Z} \times \beta \mathbf{Z})$, specifically,

$$
\left\langle M_{\beta n} T_{\alpha k} g, M_{\beta n^{\prime}} T_{\alpha k^{\prime}} \tilde{g}\right\rangle=\delta_{k k^{\prime}} \delta_{n n^{\prime}} .
$$

Hence equality holds in line (4) above, so $\alpha \beta=(\alpha \beta)^{2}$, and therefore $\alpha \beta=1$. This proves part (c) of Theorem 1.

Part (d) of Theorem 1, dealing with Riesz sequences, is also related to Wexler-Raz, and more specifically to a stronger result known as the Duality Principle (sometimes called the Ron-Shen Duality Principle). A restricted form of the Duality Principle is as follows, cf. [25, Sec. 7.4], and see [30] for more detailed discussion and references.

Theorem 4 (Duality Principle). Let $g \in L^{2}(\mathbf{R})$ and let $\Lambda=\alpha \mathbf{Z} \times \beta \mathbf{Z}$ where $\alpha, \beta>0$. Then the following statements are equivalent.

(a) $\mathcal{G}(g, \alpha \mathbf{Z} \times \beta \mathbf{Z})$ is a frame for $L^{2}(\mathbf{R})$.

(b) $\mathcal{G}\left(g, \frac{1}{\beta} \mathbf{Z} \times \frac{1}{\alpha} \mathbf{Z}\right)$ is a Riesz sequence in $L^{2}(\mathbf{R})$.

If we accept the Duality Principle, then by interchanging the roles of $\alpha, \beta$ and $\frac{1}{\beta}, \frac{1}{\alpha}$ in Theorem 4 we see that if $\mathcal{G}(g, \alpha \mathbf{Z} \times \beta \mathbf{Z})$ is a Riesz sequence in $L^{2}(\mathbf{R})$, then $\mathcal{G}\left(g, \frac{1}{\beta} \mathbf{Z} \times \frac{1}{\alpha} \mathbf{Z}\right)$ must be a frame for $L^{2}(\mathbf{R})$. But then by Theorem 1(b), we must have $\frac{1}{\beta} \frac{1}{\alpha} \leq 1$, which implies $\alpha \beta \geq 1$.

\subsection{The Density Theorem for Arbitrary Lattices}

Theorem 1 extends easily to rectangular lattices of the form $\Lambda=\alpha \mathbf{Z}^{d} \times \beta \mathbf{Z}^{d}$ in higher dimensions. We can also immediately extend the Density Theorem to certain more general lattices, specifically those of the form $\Lambda=\alpha A\left(\mathbf{Z}^{2 d}\right)$ where $A$ is a symplectic matrix and $\alpha>0$. This is because if $\Lambda$ has this form then there exists a unitary operator $\mu_{A}: L^{2}\left(\mathbf{R}^{d}\right) \rightarrow L^{2}\left(\mathbf{R}^{d}\right)$ and scalars $c_{A}(\alpha k, \beta n)$ with unit modulus such that

$$
\mathcal{G}\left(g, \alpha A\left(\mathbf{Z}^{2 d}\right)\right)=\left\{c_{A}(\alpha k, \beta n) \mu_{A}\left(M_{\alpha n} T_{\alpha k} h\right)\right\}_{k, n \in \mathbf{Z}^{d}},
$$

where $h=\mu_{A}^{-1}(g)$. The operator $\mu_{A}$ is called a metaplectic transform. The reason that symplectic matrices and metaplectic transforms arise in this context is directly related to the representation theory of the Heisenberg group, see [25, Sec. 9.4] for definitions and details.

If we remove the scalars $c_{A}(\alpha k, \beta n)$ from the righthand set in (5), then the resulting set is $\mu_{A}\left(\mathcal{G}\left(h, \alpha \mathbf{Z}^{2 d}\right)\right)$, which is the image of a rectangular lattice 
Gabor system under a unitary map. Yet although the righthand set in (5) is not precisely a Gabor system, since the property of being complete, a frame, a Riesz basis, or a Riesz sequence is preserved both by unitary mappings and by multiplication of the elements by scalars of unit modulus, we see that if $A$ is a symplectic matrix, then $\mathcal{G}\left(g, \alpha A\left(\mathbf{Z}^{2 d}\right)\right)$ is complete, a frame, a Riesz basis, or a Riesz sequence in $L^{2}\left(\mathbf{R}^{d}\right)$ if and only if the same is true of the rectangular lattice Gabor system $\mathcal{G}\left(h, \alpha \mathbf{Z}^{2 d}\right)$. This allows us to easily extend the Density Theorem to the particular case where $\Lambda=\alpha A\left(\mathbf{Z}^{2 d}\right)$ with $A$ symplectic.

The surprise is that the Density Theorem actually extends to arbitrary lattices $\Lambda=A\left(\mathbf{Z}^{2 d}\right)$, where $A$ can be any invertible $2 d \times 2 d$ matrix. This is nontrivial when $A$ is not a multiple of a symplectic matrix.

Define the volume of a lattice $A\left(\mathbf{Z}^{2 d}\right)$ to be the area of a fundamental domain for the lattice:

$$
\operatorname{vol}\left(A\left(\mathbf{Z}^{2 d}\right)\right)=|\operatorname{det}(A)| .
$$

In particular, for a one-dimensional $(d=1)$ rectangular lattice, $\operatorname{vol}(\alpha \mathbf{Z} \times$ $\beta \mathbf{Z})=\alpha \beta$. With this notation, the Density Theorem with respect to arbitrary lattices is as follows.

Theorem 5 (Density Theorem for Lattices). Let $g \in L^{2}\left(\mathbf{R}^{d}\right)$ and let $\Lambda=A\left(\mathbf{Z}^{2 d}\right)$ where $A$ is an invertible $2 d \times 2 d$ matrix. Then the following statements hold.

(a) If $\operatorname{vol}(A)>1$, then $\mathcal{G}\left(g, A\left(\mathbf{Z}^{2 d}\right)\right)$ is incomplete in $L^{2}\left(\mathbf{R}^{d}\right)$.

(b) If $\mathcal{G}\left(g, A\left(\mathbf{Z}^{2 d}\right)\right)$ is a frame for $L^{2}\left(\mathbf{R}^{d}\right)$, then $0<\operatorname{vol}(A) \leq 1$.

(c) $\mathcal{G}\left(g, A\left(\mathbf{Z}^{2 d}\right)\right)$ is a Riesz basis for $L^{2}\left(\mathbf{R}^{d}\right)$ if and only if it is a frame for $L^{2}\left(\mathbf{R}^{d}\right)$ and $\operatorname{vol}(A)=1$.

(d) If $\mathcal{G}\left(g, A\left(\mathbf{Z}^{2 d}\right)\right)$ is a Riesz sequence in $L^{2}\left(\mathbf{R}^{d}\right)$, then $\operatorname{vol}(A) \geq 1$.

We refer to [30] for a complete discussion of references.

Parts (b) and (c) of the Density Theorem are immediate consequences of Ramanathan and Steger's results on the Density Theorem for irregular Gabor frames in [47], which we will discuss in more detail below. More precisely, [47] applies to $d=1$; the extension to higher dimensions was made in [11] and is also proved in this chapter.

Parts (b), (c), and (d) are consequences of the extension by Feichtinger and Kozek of the Wexler-Raz relations and the Duality Principle to arbitrary lattices [20]. In that paper, they introduced the notion of the adjoint of a general lattice (which is distinct from the better-known dual lattice that plays a role in many formulas in Fourier analysis).

It appears that part (a) of the Density Theorem was only established in its full generality recently, by Bekka. The following is [7, Thm. 4], and is only a special case of the more general results obtained in that paper (Bekka himself attributes this result to Feichtinger and Kozek [20], but while as we have mentioned that paper does contain many results for Gabor systems on arbitrary lattices, it does not contain Theorem 6). 
Theorem 6 (Existence of Lattice Gabor Frames). Let $\Lambda=A\left(\mathbf{Z}^{2 d}\right)$ be a lattice in $\mathbf{R}^{2 d}$. Then the following statements are equivalent.

(a) $\operatorname{vol}(\Lambda) \leq 1$.

(b) There exists a $g \in L^{2}\left(\mathbf{R}^{d}\right)$ such that $\mathcal{G}(g, \Lambda)$ is complete in $L^{2}\left(\mathbf{R}^{d}\right)$.

(c) There exists a $g \in L^{2}\left(\mathbf{R}^{d}\right)$ such that $\mathcal{G}(g, \Lambda)$ is a frame for $L^{2}\left(\mathbf{R}^{d}\right)$.

The following statements are also equivalent.

(a') $\operatorname{vol}(\Lambda)=1$.

(b') There exists a $g \in L^{2}\left(\mathbf{R}^{d}\right)$ such that $\mathcal{G}(g, \Lambda)$ is a Riesz basis for $L^{2}\left(\mathbf{R}^{d}\right)$.

(c') There exists a $g \in L^{2}\left(\mathbf{R}^{d}\right)$ such that $\mathcal{G}(g, \Lambda)$ is an orthonormal basis for $L^{2}\left(\mathbf{R}^{d}\right)$.

For the case of separable lattices, i.e., $\Lambda=A\left(\mathbf{Z}^{d}\right) \times B\left(\mathbf{Z}^{d}\right)$, the equivalences in Theorem 6 were earlier proved in [28], [22].

We feel that Theorem 6 is quite surprising. For the one-dimensional case, Daubechies and Grossmann [15] conjectured that if $\varphi(t)=2^{1 / 4} e^{-\pi t^{2}}$ is the Gaussian window, then $\mathcal{G}(\varphi, \alpha \mathbf{Z} \times \beta \mathbf{Z})$ is a frame if and only if $0<\alpha \beta<1$. Evidence supporting this conjecture was given in [13], and the full conjecture was proved by Lyubarskii [42] and by Seip and Wallstén [50], [51] (see also the simple proof and additional references in [35]). It is therefore tempting to expect that if we let $\Phi(t)=2^{d / 4} e^{-\pi t \cdot t}$ be the $d$-dimensional Gaussian function, then $\mathcal{G}\left(\Phi, A\left(\mathbf{Z}^{2 d}\right)\right)$ will be a frame whenever $\operatorname{vol}(A)<1$. However, this is false. Even if we consider more general rectangular lattices of the form

$$
\Lambda=\prod_{i=1}^{d} \alpha_{i} \mathbf{Z} \times \prod_{i=1}^{d} \beta_{i} \mathbf{Z},
$$

i.e., a diagonal matrix $A$, it follows from the Lyubarskii and Seip/Wallstén characterization for $d=1$ that $\mathcal{G}(\Phi, \Lambda)$ is complete in $L^{2}\left(\mathbf{R}^{d}\right)$ if and only if $\alpha_{i} \beta_{i} \leq 1$ for each $i$. Hence if $0<\operatorname{vol}(\Lambda)<1$ but $\alpha_{i} \beta_{i}>1$ for some $i$, then $\mathcal{G}(\Phi, \Lambda)$ will be incomplete. In particular, whenever $d>1$ there exist lattices $\Lambda$ with arbitrarily small volume such that $\mathcal{G}(\Phi, \Lambda)$ is incomplete in $L^{2}\left(\mathbf{R}^{d}\right)$. Yet by Theorem 6 , there must exist some $g$ such that $\mathcal{G}(g, \Lambda)$ is a frame.

However, Bekka's proof of Theorem 6 is not constructive. On the other hand, the proof in [28] of Theorem 6 for the special case of separable lattices $\Lambda=A\left(\mathbf{Z}^{d}\right) \times B\left(\mathbf{Z}^{d}\right)$ is constructive. The window constructed in [28] is the characteristic function of a set. In some cases this set will be compact; e.g., this is the case if $A, B$ have all rational entries.

\subsection{The Density Theorem for Irregular Gabor Systems}

All of the previous discussion relied in one way or another on the structural properties of the index set $\Lambda$. However, consider what happens if we take a 
lattice $A\left(\mathbf{Z}^{2 d}\right)$ and perturb even one single point of the lattice - the resulting set $\Lambda$ is no longer a lattice. Every "algebraic" tool that has so far been mentioned, including the Duality Principle, the Wexler-Raz biorthogonality relations, the Zak transform, and von Neumann algebra techniques (and other techniques that we have not described such as the Walnut representation and the Janssen representation), is rendered inapplicable to the study of $\mathcal{G}(g, \Lambda)$.

Yet necessary conditions for $\mathcal{G}(g, \Lambda)$ to be a frame or a Riesz basis for $L^{2}\left(\mathbf{R}^{d}\right)$ are known even for arbitrary sequences $\Lambda$. These require entirely new tools, which were first supplied by H. Landau [40], Ramanathan and Steger [47], and Janssen [36], who provided the first partial extensions of the Density Theorem to irregular Gabor frames (and, in the case of [36], to more general systems).

In Theorems 1 and 5 , the value that distinguishes between the various cases is the volume of the lattice, which is the area of a fundamental domain for the lattice. In the irregular setting there is no analogue of a fundamental domain, and instead it is the Beurling density of $\Lambda$ that distinguishes between the various cases. Beurling density measures in some sense the average number of points inside unit cubes. However, because the points are not uniformly distributed, there is not a single definition, but rather lower and upper limits to the average density, which we denote by $D^{-}(\Lambda)$ and $D^{+}(\Lambda)$, respectively. The precise definition is given in Section 2.4 below.

The Density Theorem for Gabor systems with arbitrary index sets is as follows (we say that $\Lambda$ is uniformly separated if there exists a $\delta>0$ such that $|\lambda-\mu| \geq \delta$ for all $\lambda \neq \mu \in \Lambda$ ).

Theorem 7 (Density Theorem for Gabor Frames). Let $g \in L^{2}\left(\mathbf{R}^{d}\right)$ be given and let $\Lambda$ be a sequence of points in $\mathbf{R}^{2 d}$.

(a) If $\mathcal{G}(g, \Lambda)$ is complete in $L^{2}\left(\mathbf{R}^{d}\right)$, then $0 \leq D^{-}(\Lambda) \leq D^{+}(\Lambda) \leq \infty$.

(b) If $\mathcal{G}(g, \Lambda)$ is a frame for $L^{2}\left(\mathbf{R}^{d}\right)$, then $1 \leq D^{-}(\Lambda) \leq D^{+}(\Lambda)<\infty$.

(c) If $\mathcal{G}(g, \Lambda)$ is a Riesz basis for $L^{2}\left(\mathbf{R}^{d}\right)$, then $D^{-}(\Lambda)=D^{+}(\Lambda)=1$. Moreover, $\Lambda$ is uniformly separated.

(d) If $\mathcal{G}(g, \Lambda)$ is a Riesz sequence in $L^{2}\left(\mathbf{R}^{d}\right)$, then $0 \leq D^{-}(\Lambda) \leq D^{+}(\Lambda) \leq 1$. Moreover, $\Lambda$ is uniformly separated.

The critical value $D^{ \pm}(\Lambda)=1$ is sometimes called the Nyquist density. .

To compare to the previous Density Theorems, we note that the Beurling density of a rectangular lattice is

$$
D^{-}\left(\alpha \mathbf{Z}^{d} \times \beta \mathbf{Z}^{d}\right)=D^{+}\left(\alpha \mathbf{Z}^{d} \times \beta \mathbf{Z}^{d}\right)=\frac{1}{(\alpha \beta)^{d}},
$$

and the density of a general lattice $A\left(\mathbf{Z}^{2 d}\right)$ is

$$
D^{-}\left(A\left(\mathbf{Z}^{2 d}\right)\right)=D^{+}\left(A\left(\mathbf{Z}^{2 d}\right)\right)=\frac{1}{|\operatorname{det}(A)|}=\frac{1}{\operatorname{vol}(A)},
$$


i.e., high density corresponds to small volume and conversely.

There is a rich literature on ideas and results closely related to the Density Theorem in the settings of sampling and interpolation of band-limited functions, density conditions for systems of windowed exponentials, sampling in the Bargmann-Fock space of entire functions, and density conditions for abstract localized frames. Indeed, the precise formulation of the Nyquist density is due to Landau [38], [39], in the context of sampling and interpolation of band-limited functions. We will not attempt to describe that literature here.

\subsection{A Brief History}

Note that in contrast to the Density Theorem for the case of lattices, part (a) of Theorem 7 has an empty conclusion (i.e., it gives no information), for by definition of Beurling density we always have that $0 \leq D^{-}(\Lambda) \leq D^{+}(\Lambda) \leq \infty$. Ramanathan and Steger conjectured in [47] that Theorem 7(a) should be improvable to say that if $D^{+}(\Lambda)<1$, then $\mathcal{G}(g, \Lambda)$ is incomplete in $L^{2}(\mathbf{R})$. This was shown in [8] to be false: for any $\varepsilon>0$ there exists a function $g \in L^{2}(\mathbf{R})$ and a sequence $\Lambda \subset \mathbf{R}^{2}$ with $0<D^{+}(\Lambda)<\varepsilon$ such that $\mathcal{G}(g, \Lambda)$ is complete. Indeed, it has even been shown that there exist $g$ and $\Lambda$ such that $D^{+}(\Lambda)=0$ yet $\mathcal{G}(g, \Lambda)$ is complete [44], [45], [49]. Thus there is a stark contrast between lattice and non-lattice Gabor systems with regard to completeness.

For the one-dimensional case $(d=1)$, part (b) of Theorem 7 was first proved, but with extra hypotheses on $g$, by H. Landau [40]. Landau's technique is related to the energy concentration result for rectangular lattice Gabor frames proved by Daubechies [14, Thm. 3.5.2].

Parts (b) and (c) were proved for arbitrary $g \in L^{2}(\mathbf{R})$, but with extra hypotheses on $\Lambda$, by Ramanathan and Steger [47]. Specifically, Ramanathan and Steger only considered the case where $\Lambda$ is uniformly separated. However, not all irregular Gabor frames are uniformly separated. For example, if we let $g=\chi_{[0,1]}$, then $\mathcal{G}\left(g, \mathbf{Z}^{2} \cup \sqrt{2} \mathbf{Z}^{2}\right)$ is a frame, but the index set is not uniformly separated. The fact a Gabor Riesz sequence must be uniformly separated was proved in [18].

Janssen [36] proved part (b) for "half irregular" $\Lambda \subset \mathbf{R}^{2}$, i.e., of the form $\Lambda=\alpha \mathbf{Z} \times \Gamma$ with $\Gamma$ irregular. Furthermore, Janssen's result actually applies to certain more general systems whose elements need not be exact time-frequency shifts of a single generator.

In their paper, Ramanathan and Steger introduced a fundamental new concept in the study of Gabor frames. Namely, they showed that all Gabor frames satisfy a certain Homogeneous Approximation Property (HAP). Gröchenig and Razafinjatovo [27] modified and improved this technique to derive density conditions in the context of sampling of bandlimited functions. Inspired by [27], a complete proof of Theorem 7, without restrictions on $g$ or $\Lambda$, was given in [11]. Additionally, the Density Theorem was extended in [11] to the case of higher dimensions and finitely many generators, and some other applications were made. Further results related to the density of systems of 
windowed exponentials appear in [31], and the role of the HAP in wavelet theory is expored in [32].

The HAP was one of the inspirations for the study of abstract localized frames in the papers [4], [5]. Among other results, it was shown there that the HAP and the Density Theorem are consequence of more general considerations rather than the particular rigid structure of Gabor systems. In particular, part (d) of Theorem 7 was first proved in [5]. Localized frames were independently introduced and published by Gröchenig [26], for entirely different purposes.

\subsection{Goals of This Paper}

The introduction of the HAP by Ramanathan and Steger was a fundamental advance in the understanding of arbitrary ("irregular") Gabor frames. Unfortunately, the proofs in [47] were mostly given in "sketch" form, and the significance of this paper has not generally been well-appreciated. Our goal in the remainder of this chapter is to give a more complete exposition of some of the proofs of the results of Ramanathan and Steger. In the process, we also show that their approach can in fact be applied to completely arbitrary Gabor frames $\mathcal{G}(g, \Lambda)$, without restrictions on $g$ or $\Lambda$ (although the modified technique introduced by Gröchenig and Razafinjatovo in [27] clearly remains more "elegant"). We also extend to the case of finitely many generators and to higher dimensions. The theorems we obtain are not new, and indeed are contained in full generality in [11]. However, we hope this chapter will inspire the reader to further consider the Homogeneous Approximation Property and to pursue the most recent results on localized frames.

Of course, [47] is not the only significant advance in the theory of irregular Gabor frames. An incomplete list of other references not already mentioned above includes [24], [12], [52], [3], [41], [53], [37].

Our chapter is organized as follows. In Section 2 we present our notation, background, and technical results on the short-time Fourier transform, frames, density, and weak convergence of sequences of sets. The drawback of the Ramanathan and Steger approach is evident in this section - a great deal

of technicality is required to deal with the weak convergence of sequences of sequences and their corresponding Gabor systems. The modified HAP introduced by Gröchenig and Razafinjatovo allows a much "cleaner" approach, and in particular, the use of weak convergence is avoided in [27]. In Section 3 we define several versions of the HAP, and use this to derive the Density Theorem in higher dimensions with finitely many generators.

\section{Notation and Preliminaries}

In this section we define our terminology and provide some background and discussion related to our results. 


\subsection{General notation}

Let $\Lambda=\left\{\lambda_{i}\right\}_{i \in I}$ be a sequence of points in $\mathbf{R}^{2 d}$, with a countable index set $I$. For simplicity of notation, we will write $\Lambda \subset \mathbf{R}^{2 d}$, but we always mean that $\Lambda$ is a sequence and not merely a subset of $\mathbf{R}^{2 d}$. In particular, repetitions of elements are allowed. A sequence $\Lambda$ is a lattice if $\Lambda=A\left(\mathbf{Z}^{2 d}\right)$ where $A$ is a $2 d \times 2 d$ invertible matrix.

Often we will deal with several sequences $\Lambda_{1}, \ldots, \Lambda_{N} \subset \mathbf{R}^{d}$, and will use the notation $\Lambda=\bigcup_{k=1}^{N} \Lambda_{k}$ to denote the disjoint union of these sequences. In particular, if each $\Lambda_{k}$ is indexed as $\Lambda_{k}=\left\{\lambda_{j k}\right\}_{j \in \mathbf{N}}$, then $\Lambda$ is the sequence $\Lambda=\left\{\lambda_{11}, \ldots, \lambda_{1 N}, \lambda_{21}, \ldots, \lambda_{2 N}, \ldots\right\}$.

Given $z \in \mathbf{R}^{2 d}$ and $h>0$, we let $Q_{h}(z)$ denote the closed cube in $\mathbf{R}^{2 d}$ centered at $z$ with side lengths $h$, specifically,

$$
Q_{h}(z)=\prod_{j=1}^{2 d}\left[z_{j}-\frac{h}{2}, z_{j}+\frac{h}{2}\right] .
$$

If $H$ is a Hilbert space and $f_{i} \in H$ for $i \in I$, then $\operatorname{span}\left\{f_{i}\right\}_{i \in I}$ will denote the finite linear span of $\left\{f_{i}\right\}_{i \in I}$, and $\overline{\operatorname{span}}\left\{f_{i}\right\}_{i \in I}$ will denote the closure of this set in $H$. The distance from a vector $f \in H$ to a closed subspace $V \subset H$ is $\operatorname{dist}(f, V)=\inf \{\|f-v\|: v \in V\}=\left\|f-P_{V} f\right\|$, where $P_{V}$ is the orthogonal projection onto $V$.

The translation of a function $g$ by $x \in \mathbf{R}^{d}$ is $T_{x} g(t)=g(t-x)$, and the modulation of $g$ by $\xi \in \mathbf{R}^{d}$ is $M_{\xi} g(t)=e^{2 \pi i \xi \cdot t} g(t)$. Using this notation, $\mathcal{G}(g, \Lambda)=\left\{M_{\xi} T_{x} g\right\}_{(x, \xi) \in \Lambda}$.

The family of translation operators is strongly continuous, as is the family of modulation operators. This implies the following.

Lemma 8. Let $f \in L^{2}\left(\mathbf{R}^{d}\right)$ and $\varepsilon>0$ be given. Then there exists $\delta>0$ such that

$$
\forall u, \eta \in \mathbf{R}^{d}, \quad|u|,|\eta|<\delta \Longrightarrow\left\|M_{\eta} T_{u} f-f\right\|_{2}<\varepsilon .
$$

Corollary 9. Let $K \subset \mathbf{R}^{d}$ be compact, and let $f \in L^{2}\left(\mathbf{R}^{d}\right)$ and $\varepsilon>0$ be given. Then there exists $\delta>0$ such that

$\forall(x, \xi) \in K, \forall(u, \eta) \in \mathbf{R}^{2 d}, \quad|u|,|\eta|<\delta \Longrightarrow\left\|M_{\eta} T_{u} M_{\xi} T_{x} f-M_{\xi} T_{x} f\right\|_{2}<\varepsilon$.

\subsection{Amalgam Space Properties of the Short-Time Fourier Transform}

We will need the following facts regarding properties of the short-time Fourier transform. We refer to [25] for a detailed discussion of the STFT, the modulation spaces, and the amalgam spaces, and for references to the original literature. Also, a survey of amalgam spaces appears in [29]. 
Definition 10. The short-time Fourier transform (STFT) of a function $f \in$ $L^{2}\left(\mathbf{R}^{d}\right)$ with respect to a window $g \in L^{2}\left(\mathbf{R}^{d}\right)$ is

$$
V_{g} f(x, \xi)=\left\langle f, M_{\xi} T_{x} g\right\rangle=\int_{\mathbf{R}^{d}} f(t) e^{-2 \pi i \xi \cdot t} \overline{g(t-x)} d t, \quad(x, \xi) \in \mathbf{R}^{2 d} .
$$

We have that $V_{g} f$ is continuous and bounded on $\mathbf{R}^{2 d}$, and

$$
\left\|V_{g} f\right\|_{2}=\|f\|_{2}\|g\|_{2}
$$

see [25, Cor. 3.2.2]. We will need some finer properties of the STFT, which we recall next.

The modulation spaces were invented by Feichtinger, and extensively investigated by Feichtinger and Gröchenig. They are now recognized as the appropriate function spaces for time-frequency analysis, and occur naturally in mathematical problems involving time-frequency shifts $M_{\xi} T_{x}$. For our purposes, the following particular modulation space will be sufficient.

Definition 11. The modulation space $M^{1}\left(\mathbf{R}^{d}\right)$ consists of all $f \in L^{2}\left(\mathbf{R}^{d}\right)$ such that

$$
\|f\|_{M^{1}}=\left\|V_{\gamma} f\right\|_{1}=\int_{\mathbf{R}^{d}} \int_{\mathbf{R}^{d}}\left|V_{\gamma} f(x, \xi)\right| d x d \xi<\infty,
$$

where $\gamma(t)=2^{d / 4} e^{-\pi t \cdot t}$.

$M^{1}$ is a Banach space, and its definition is independent of the choice of window, i.e., $\gamma$ may be replaced by any nonzero function in the Schwartz class, or indeed by any function in $M^{1}$, in the sense of equivalent norms. $M^{1}$ contains the Schwartz class, and hence is dense in $L^{2}$. The space $M^{1}$ is also called the Feichtinger algebra, and is sometimes denoted $S_{0}$.

We will need the following amalgam space property of the STFT, see [25, Thm. 12.2.1].

Lemma 12. If $g \in L^{2}\left(\mathbf{R}^{d}\right)$ and $f \in M^{1}\left(\mathbf{R}^{d}\right)$, then $V_{g} f$ belongs to the amalgam space $W\left(L^{\infty}, \ell^{2}\right)$, i.e.,

$$
\left\|V_{g} f\right\|_{W\left(L^{\infty}, \ell^{2}\right)}=\sum_{j \in \mathbf{Z}^{2 d}}\left\|V_{g} f \cdot \chi_{Q_{1}(j)}\right\|_{\infty}^{2}<\infty .
$$

Any size cubes may be used to define the amalgam space (in the sense of equivalent norms), i.e., we may replace the unit cubes $Q_{1}(j)$ in (6) by the cubes $Q_{\delta}(j \delta)$ where $\delta>0$.

The first amalgam spaces were introduced by Wiener in his study of generalized harmonic analysis. A comprehensive general theory of amalgam spaces on locally compact groups was introduced by Feichtinger, and extensively studied by Feichtinger and Gröchenig. 
The amalgam space properties of the STFT were not available when Ramanathan and Steger wrote their paper. Instead, they made use of the fact that if $\gamma$ is the Gaussian function $\gamma(t)=e^{-t^{2}}$, then the STFT $V_{\gamma} f$ is very closely related to the Bargmann transform $B f$, which is an analytic function. Our use of amalgam spaces is perhaps a little more straightforward, but is not an essential change to their argument.

\subsection{Bases and Frames}

We use the standard notations for frames and Riesz bases as found in the texts [10], [14], [25], [54] or the research-tutorial [33].

Definition/Facts 13. Let $\mathcal{F}=\left\{f_{i}\right\}_{i \in \mathbf{N}}$ be a sequence in a Hilbert space $H$.

(a) $\mathcal{F}$ is complete if its finite linear span is dense in $H$.

(b) $\mathcal{F}$ is minimal if there exists a sequence $\left\{\tilde{f}_{i}\right\}_{i \in \mathbf{N}}$ in $H$ that is biorthogonal to $\mathcal{F}$, i.e., $\left\langle f_{i}, \tilde{f}_{j}\right\rangle=\delta_{i j}$ for $i, j \in \mathbf{N}$. Equivalently, $\left\{f_{i}\right\}_{i \in \mathbf{N}}$ is minimal if $f_{j} \notin \overline{\operatorname{span}}\left\{f_{i}\right\}_{i \neq j}$ for each $j \in \mathbf{N}$. We say that $\mathcal{F}$ is exact if it is both minimal and complete. In this case the biorthogonal sequence is unique.

(c) A Riesz basis is the image of an orthonormal basis under a continuous invertible mapping of $H$ onto itself.

(d) $\mathcal{F}$ is a frame for $H$ if there exist constants $A, B>0$, called frame bounds, such that

$$
\forall f \in H, \quad A\|f\|^{2} \leq \sum_{i=1}^{\infty}\left|\left\langle f, f_{i}\right\rangle\right|^{2} \leq B\|f\|^{2} .
$$

All Riesz bases are frames, but not conversely.

(e) If $\mathcal{F}$ satisfies at least the second inequality in (7) then we say that it is a Bessel sequence or that it possesses an upper frame bound, and we call $B$ $a$ Bessel bound. We have that $\mathcal{F}$ is a Bessel sequence if and only if the analysis operator $C f=\left\{\left\langle f, f_{i}\right\rangle\right\}_{i \in \mathbf{N}}$ is a bounded mapping $C: H \rightarrow \ell^{2}$. In this case, the adjoint of $C$ is the synthesis operator $C^{*}: \ell^{2} \rightarrow H$ given by $C^{*}\left(\left\{c_{i}\right\}_{i \in \mathbf{N}}\right)=\sum c_{i} f_{i}$ (the series converges unconditionally in the norm of $H)$. In particular, if $B$ is a Bessel bound then

$$
\forall\left\{c_{i}\right\}_{i \in \mathbf{N}} \in \ell^{2}, \quad\left\|\sum_{i \in \mathbf{N}} c_{i} f_{i}\right\|^{2} \leq B \sum_{i \in \mathbf{N}}\left|c_{i}\right|^{2} .
$$

Hence $\left\|f_{i}\right\|^{2} \leq B$ for every $i$, so every Bessel sequence is uniformly bounded above in norm.

(f) If $\mathcal{F}$ is a frame then the frame operator $S f=C^{*} C f=\sum\left\langle f, f_{i}\right\rangle f_{i}$ is a bounded, positive definite, invertible map of $H$ onto itself. 
(g) Every frame $\mathcal{F}$ has a canonical dual frame $\tilde{\mathcal{F}}=\left\{\tilde{f}_{i}\right\}_{i \in \mathbf{N}}$ given by $\tilde{f}_{i}=$ $S^{-1} f_{i}$ where $S$ is the frame operator. Writing out and rearranging the equalities $f=S S^{-1} f=S^{-1} S f$ gives the frame expansions

$$
\forall f \in H, \quad f=\sum_{i=1}^{\infty}\left\langle f, \tilde{f}_{i}\right\rangle f_{i}=\sum_{i=1}^{\infty}\left\langle f, f_{i}\right\rangle \tilde{f}_{i},
$$

and furthermore the series in (9) converge unconditionally for every $f$ (so any countable index set can be used to index a frame). In general, the coefficients in (9) need not be unique.

(h) If $\mathcal{F}$ is a frame and $\tilde{\mathcal{F}}$ is its canonical dual frame then the following statements are equivalent:
i. $\mathcal{F}$ is a Riesz basis,
ii. $\mathcal{F}$ is exact,
iii. the coefficients in (9) are unique for each $f \in H$, iv. $\mathcal{F}$ and $\tilde{\mathcal{F}}$ are biorthogonal.

(i) We say that $\mathcal{F}$ is a frame sequence or a Riesz sequence if it is a frame or a Riesz basis for its closed linear span in $H$, respectively.

For the case of a Gabor frame $\mathcal{G}(g, \Lambda)$, if $\Lambda$ is a rectangular lattice of the form $\Lambda=\alpha \mathbf{Z}^{d} \times \beta \mathbf{Z}^{d}$, then the canonical dual frame is also a Gabor frame with respect to the same lattice, i.e., it has the form $\mathcal{G}(\tilde{g}, \Lambda)$ where $\tilde{g}=S^{-1} g$ [25, Prop. 5.2.1]. However, if $\Lambda$ is not a lattice then the dual frame will not be a Gabor frame in general. There will exist a canonical dual frame, but this dual frame need not consist of time-frequency shifts of a single function.

\subsection{Beurling Density}

Beurling density measures in some sense the average number of points contained in unit cubes. The precise definition is as follows.

Definition 14. Let $\Lambda=\left\{\lambda_{i}\right\}_{i \in I}$ be a sequence of points in $\mathbf{R}^{2 d}$. The upper and lower Beurling densities of $\Lambda$ are, respectively,

$$
\begin{aligned}
& D^{+}(\Lambda)=\limsup _{h \rightarrow \infty} \sup _{z \in \mathbf{R}^{d}} \frac{\#\left(\Lambda \cap Q_{h}(z)\right)}{h^{2 d}}, \\
& D^{-}(\Lambda)=\liminf _{h \rightarrow \infty} \inf _{z \in \mathbf{R}^{d}} \frac{\#\left(\Lambda \cap Q_{h}(z)\right)}{h^{2 d}} .
\end{aligned}
$$

In general

$$
\sum_{k=1}^{N} D^{-}\left(\Lambda_{k}\right) \leq D^{-}\left(\bigcup_{k=1}^{N} \Lambda_{k}\right) \leq D^{+}\left(\bigcup_{k=1}^{N} \Lambda_{k}\right) \leq \sum_{k=1}^{N} D^{+}\left(\Lambda_{k}\right),
$$


but these inequalities may be strict, e.g., consider

$\Lambda_{1}=\left\{\left(k_{1}, k_{2}\right) \in \mathbf{Z}^{2}: k_{1} \geq 0, k_{2} \in \mathbf{Z}\right\}, \quad \Lambda_{2}=\left\{\left(k_{1}, k_{2}\right) \in \mathbf{Z}^{2}: k_{1}<0, k_{2} \in \mathbf{Z}\right\}$.

We say that a sequence $\Lambda$ is $\delta$-uniformly separated if $|\lambda-\mu| \geq \delta$ for all $\lambda \neq \mu \in \Lambda$.

A sequence $\Lambda$ satisfies $D^{+}(\Lambda)<\infty$ if and only if $\Lambda=\Lambda_{1} \cup \cdots \cup \Lambda_{N}$ with each $\Lambda_{k}$ being $\delta_{k}$-uniformly separated for some $\delta_{k}>0$ [11, Lem. 2.3].

It was shown in [11] that if $\mathcal{G}(g, \Lambda)$ is a Bessel sequence, then $\Lambda$ must have finite density. Specifically, we have the following.

Proposition 15. If $g \in L^{2}\left(\mathbf{R}^{d}\right) \backslash\{0\}$ and $\Lambda \subset \mathbf{R}^{2 d}$ are such that $\mathcal{G}(g, \Lambda)$ is a Bessel sequence, then $D^{+}(\Lambda)<\infty$.

Ramanathan and Steger only considered uniformly separated sequences, which automatically have finite density.

\subsection{Weak Convergence of Sequences}

In this section we develop some machinery concerning the weak convergence of a sequence of subsets of $\mathbf{R}^{2 d}$.

Definition 16. (a) Given a set $E \subset \mathbf{R}^{2 d}$, for each $t \geq 0$ define

$$
E_{t}=\left\{x \in \mathbf{R}^{2 d}: \operatorname{dist}(x, E)<t\right\} .
$$

(b) The Fréchet distance between two closed sets $E, F \subset \mathbf{R}^{2 d}$ is

$$
[E, F]=\inf \left\{t \geq 0: E \subset F_{t} \text { and } F \subset E_{t}\right\} .
$$

(c) Given closed sets $E_{n} \subset \mathbf{R}^{2 d}$ and given a closed set $F \subset \mathbf{R}^{2 d}$, we say that $E_{n}$ converges weakly to $F$ if

$$
\forall \text { compact } K \subset \mathbf{R}^{2 d}, \quad \lim _{n \rightarrow \infty}\left[E_{n} \cap K, F \cap K\right]=0 .
$$

In this case we write $E_{n} \stackrel{\mathrm{w}}{\longrightarrow} F$.

Lemma 17. Let $\Lambda_{1}, \ldots, \Lambda_{N} \subset \mathbf{R}^{2 d}$ be countable sequences such that each $\Lambda_{k}$ is $\delta_{k}$-uniformly separated for some $\delta_{k}>0$. Then given any sequence of points $\left\{z_{n}\right\}_{n \in \mathbf{N}}$ in $\mathbf{R}^{2 d}$, there exists a subsequence $\left\{w_{n}\right\}_{n \in \mathbf{N}}$ of $\left\{z_{n}\right\}_{n \in \mathbf{N}}$ and there exist sequences $\Lambda_{k}^{\prime} \subset \mathbf{R}^{2 d}$ such that

$$
\Lambda_{k}-w_{n} \stackrel{\mathrm{w}}{\longrightarrow} \Lambda_{k}^{\prime} \text { as } n \rightarrow \infty, \quad k=1, \ldots, N .
$$

Further, if for some $k$ we have $D^{-}\left(\Lambda_{k}\right)>0$, then we can construct $\Lambda_{k}^{\prime}$ so that $D^{-}\left(\Lambda_{k}^{\prime}\right)>0$ as well. 
Proof. Since a subsequence of a weakly convergent sequence of sets is still weakly convergent to the same limit set, it suffices to consider the case $N=1$. Thus, assume that $\Lambda \subset \mathbf{R}^{2 d}$ is $\delta$-uniformly separated and that $\left\{z_{n}\right\}_{n \in \mathbf{N}}$ is given.

If $D^{-}(\Lambda)>0$, there will exist some $r>0$ such that every cube $Q_{r}(z)$ in $\mathbf{R}^{2 d}$ will contain at least one element of $\Lambda$. If $D^{-}(\Lambda)=0$, define $r=1$. Write

$$
\mathbf{R}^{2 d}=\bigcup_{j \in \mathbf{N}} R_{j}
$$

where the $R_{j}$ are closed cubes of sidelength $r$ with disjoint interiors.

Since $\Lambda$ is uniformly separated, there is a finite integer $M$ such that

$$
\forall j, n \in \mathbf{N}, \quad \#\left(\Lambda-z_{n} \cap R_{j}\right) \leq M .
$$

Furthermore, in the case that $D^{-}(\Lambda)>0$ we also have

$$
\forall j, n \in \mathbf{N}, \quad 1 \leq \#\left(\Lambda-z_{n} \cap R_{j}\right) .
$$

Because of equation (10), there must exist some integer $0 \leq M_{1} \leq M$ and some subsequence $\left\{z_{n}^{1}\right\}_{n \in \mathbf{N}}$ of $\left\{z_{n}\right\}_{n \in \mathbf{N}}$ such that

$$
\forall n \in \mathbf{N}, \quad \#\left(\Lambda-z_{n}^{1} \cap R_{1}\right)=M_{1} .
$$

In the case that $D^{-}(\Lambda)>0$, equation (11) implies that $M_{1} \geq 1$.

Similarly, again because of equation (10), there must exist some integer $0 \leq M_{2} \leq M$ and some subsequence $\left\{z_{n}^{2}\right\}_{n \in \mathbf{N}}$ of $\left\{z_{n}^{1}\right\}_{n \in \mathbf{N}}$ such that

$$
\forall n \in \mathbf{N}, \quad \#\left(\Lambda-z_{n}^{2} \cap R_{2}\right)=M_{2},
$$

and again $M_{2} \geq 1$ if $D^{-}(\Lambda)>0$.

Continue in this way to create a subsequence $\left\{z_{n}^{j+1}\right\}_{n \in \mathbf{N}}$ of $\left\{z_{n}^{j}\right\}_{n \in \mathbf{N}}$ for each $j \in \mathbf{N}$. Set $w_{n}=z_{n}^{n}$. Then $\left\{w_{n}\right\}_{n \in \mathbf{N}}$ is a subsequence of $\left\{z_{n}^{j}\right\}_{n \geq j}$ for each $j \in \mathbf{N}$, so

$$
\forall j \in \mathbf{N}, \quad \forall n \geq j, \quad \#\left(\Lambda-w_{n} \cap R_{j}\right)=M_{j},
$$

where $0 \leq M_{j} \leq M$.

Let $J=\left\{j \in \mathbf{N}: M_{j}>0\right\}$. For $j \in J$ and $n \geq j$, write

$$
\Lambda-w_{n} \cap R_{j}=\left\{\lambda_{j, 1}^{n}, \ldots, \lambda_{j, M_{j}}^{n}\right\} .
$$

Fix $j \in J$ and $1 \leq m \leq M_{j}$. The sequence $\left\{\lambda_{j, m}^{n}\right\}_{n \geq j}$ is contained in the compact set $R_{j}$, so it must have a convergent subsequence. By using another diagonalization argument, passing to a subsequence of $\left\{w_{n}\right\}_{n \in \mathbf{N}}$ if necessary, we can assume that $\left\{\lambda_{j, m}^{n}\right\}_{n \in \mathbf{N}}$ converges for each $j \in J$ and $m=1, \ldots, M_{j}$. Set

$$
\lambda_{j, m}=\lim _{n \rightarrow \infty} \lambda_{j, m}^{n}
$$


and define

$$
\Lambda^{\prime}=\left\{\lambda_{j, m}\right\}_{j \in J, m=1, \ldots, M_{j}} .
$$

Then for any compact set $K \subset \mathbf{R}^{2 d}$ and any $\varepsilon>0$, we have that for all $n$ large enough, every point of $\Lambda^{\prime} \cap Q$ is within $\varepsilon$ of a point in $\Lambda-w_{n}$ and conversely. That is, $\left[\Lambda-w_{n} \cap K, \Lambda^{\prime} \cap K\right]<\varepsilon$ for all $n$ large enough, so $\Lambda-w_{n} \stackrel{\mathrm{w}}{\longrightarrow} \Lambda^{\prime}$.

Finally, if $D^{-}(\Lambda)>0$, then $M_{j} \geq 1$ for every $j$, so each $R_{j}$ contains at least one element of $\Lambda^{\prime}$. Thus in this case we have $D^{-}\left(\Lambda^{\prime}\right)>0$.

Remark 18. $\Lambda^{\prime}$ could be empty. For example, consider $\Lambda=\{0\} \times \mathbf{Z}$ and $z_{n}=$ $(n, 0)$. Then $\Lambda-z_{n} \stackrel{\mathrm{w}}{\longrightarrow} \emptyset$. However, if $D^{-}(\Lambda)>0$, then Lemma 17 implies that we can construct a nonempty $\Lambda^{\prime}$.

\subsection{Weak Convergence of Gabor Frames}

We will need the following technical result.

Lemma 19. Let $g_{1}, \ldots, g_{N} \in L^{2}\left(\mathbf{R}^{d}\right) \backslash\{0\}$ and $\Lambda_{1}, \ldots, \Lambda_{N} \subset \mathbf{R}^{2 d}$ be such that:

(a) each $\Lambda_{k}$ is $\delta_{k}$-uniformly separated for some $\delta_{k}>0$, and

(b) $\mathcal{G}=\bigcup_{k=1}^{N} \mathcal{G}\left(g_{k}, \Lambda_{k}\right)$ is a frame for $L^{2}\left(\mathbf{R}^{d}\right)$ with frame bounds $A, B$.

Suppose that $z_{n} \in \mathbf{R}^{2 d}$ and $\Lambda_{1}^{\prime}, \ldots, \Lambda_{N}^{\prime} \subset \mathbf{R}^{2 d}$ are such that

$$
\Lambda_{k}-z_{n} \stackrel{\mathrm{w}}{\longrightarrow} \Lambda_{k}^{\prime} \text { as } n \rightarrow \infty, \quad k=1, \ldots, N .
$$

Then $\mathcal{G}^{\prime}=\bigcup_{k=1}^{N} \mathcal{G}\left(g_{k}, \Lambda_{k}^{\prime}\right)$ is a frame for $L^{2}\left(\mathbf{R}^{d}\right)$ with frame bounds $A, B$.

Proof. Set $\delta=\min \left\{\delta_{1} / 4, \ldots, \delta_{N} / 4\right\}$, so each $\Lambda_{k}$ is $2 \delta$-uniformly separated. In particular, any cube $Q_{\delta}(z)$ can contain at most one point of any $\Lambda_{k}$.

Choose any $\varepsilon>0$. Fix any nonzero $f \in M^{1}\left(\mathbf{R}^{d}\right)$. Then by Lemma 12 , we have $V_{g_{k}} f \in W\left(L^{\infty}, \ell^{2}\right)$. Hence we can find an $m \in \mathbf{N}$ such that

$$
\sum_{j \in \mathbf{Z}^{2 d} \backslash Q_{m}(0)}\left\|V_{g_{k}} f \cdot \chi_{Q_{\delta}(\delta j)}\right\|_{\infty}^{2}<\frac{\varepsilon}{2 N}, \quad k=1, \ldots, N .
$$

Set $R=(2 m+1) \delta$.

Since each translated set $\Lambda_{k}-z_{n}$ has the same density, we have

$$
D=\sup _{n \in \mathbf{N}, k=1, \ldots, N} \#\left(\Lambda_{k}-z_{n} \cap Q_{R}(0)\right)<\infty .
$$

Fix $\eta<\delta / 2$. Since $\Lambda_{k}-z_{n} \stackrel{\mathrm{w}}{\longrightarrow} \Lambda_{k}^{\prime}$, for compact set $K \subset \mathbf{R}^{2 d}$ we can find an $n$ such that

$$
\left[\Lambda_{k}-z_{n} \cap K, \Lambda^{\prime} \cap K\right]<\eta, \quad k=1, \ldots, N .
$$


Hence each point of $\Lambda_{k}-z_{n} \cap K$ is within $\eta$ of a point in $\Lambda^{\prime} \cap K$, and conversely. Since $\Lambda_{k}-z_{n}$ is $2 \delta$-uniformly separated and $\eta<\delta / 2$, it follows that each point of $\Lambda_{k}-z_{n} \cap K$ is within $\eta$ of a unique point in $\Lambda^{\prime} \cap K$, and conversely.

Hence, if $\lambda \neq \mu \in \Lambda^{\prime} \cap K$, then there exist unique points $\kappa \neq \nu \in \Lambda_{k}-z_{n} \cap K$ such that $|\lambda-\kappa|<\eta$ and $|\mu-\nu|<\eta$. Since $\Lambda_{k}-z_{n} \cap K$ is $2 \delta$-uniformly separated, we therefore have

$$
2 \delta \leq|\kappa-\nu| \leq|\lambda-\mu|+|\kappa-\lambda|+|\mu-\nu|<|\lambda-\mu|+\eta+\eta<|\lambda-\mu|+\delta .
$$

Thus $|\lambda-\mu|>\delta$, and hence we have that $\Lambda_{k}^{\prime} \cap K$ is $\delta$-uniformly separated. Since this is true for all compact sets $K$, we conclude that $\Lambda_{k}^{\prime}$ is $\delta$-uniformly separated. Thus each cube $Q_{\delta}(z)$ can contain at most one point of each $\Lambda_{k}^{\prime}$.

Now, if $(x, \xi) \in \mathbf{R}^{2 d} \backslash Q_{R}(0)$, then there is a unique $j \in \mathbf{Z}^{2 d} \backslash Q_{m}(0)$ such that $(x, \xi) \in Q_{\delta}(\delta j)$. Hence,

$$
\begin{aligned}
\sum_{k=1}^{N} \sum_{(x, \xi) \in \Lambda_{k}^{\prime} \backslash Q_{R}(0)}\left|\left\langle f, M_{\xi} T_{x} g\right\rangle\right|^{2} & =\sum_{k=1}^{N} \sum_{(x, \xi) \in \Lambda_{k}^{\prime} \backslash Q_{R}(0)}\left|V_{g_{k}} f(x, \xi)\right|^{2} \\
& \leq \sum_{k=1}^{N} \sum_{j \in \mathbf{Z}^{2 d} \backslash Q_{m}(0)} \sup _{(u, \eta) \in Q_{\delta}(\delta j)}\left|V_{g_{k}} f(u, \eta)\right|^{2} \\
& <\frac{\varepsilon}{2},
\end{aligned}
$$

the last inequality following from equation (12).

Similarly, for each $n \in \mathbf{N}$ we have

$$
\sum_{k=1}^{N} \sum_{(x, \xi) \in \Lambda_{k}-z_{n} \backslash Q_{R}(0)}\left|\left\langle f, M_{\xi} T_{x} g_{k}\right\rangle\right|^{2}<\frac{\varepsilon}{2} .
$$

Consider for the moment the case $D>0$. Since $Q_{R}(0)$ is compact, we have by Corollary 9 that there exists $\theta>0$ such that

$\forall(x, \xi) \in Q_{R}(0), \forall|u|,|\eta|<\theta, \quad\left\|M_{\eta} T_{u} M_{\xi} T_{x} f-M_{\xi} T_{x} f\right\|_{2}<\frac{\varepsilon}{2(N D)^{1 / 2}\|f\|_{2}}$.

Let $n$ be large enough that

$$
\left[\Lambda_{k}-z_{n} \cap Q_{R}(0), \Lambda_{k}^{\prime} \cap Q_{R}(0)\right]<\theta .
$$

Then for each $(x, \xi) \in \Lambda_{k}-z_{n} \cap Q_{R}(0)$, there exist unique points $|u(x, \xi, k)|$, $|\eta(x, \xi, k)|<\theta$ such that $(x+u(x, \xi, k), \xi+\eta(x, \xi, k)) \in \Lambda_{k}^{\prime}$. Furthermore,

$$
(x, \xi) \mapsto(x+u(x, \xi, k), \xi+\eta(x, \xi, k))
$$

is a bijection of $\Lambda_{k}-z_{n} \cap Q_{R}(0)$ onto $\Lambda_{k}^{\prime} \cap Q_{R}(0)$. Hence, 


$$
\begin{aligned}
& \mid\left(\sum_{k=1}^{N} \sum_{(x, \xi) \in \Lambda_{k}^{\prime} \cap Q_{R}(0)}\left|\left\langle f, M_{\xi} T_{x} g\right\rangle\right|^{2}\right)^{1 / 2} \\
& \quad-\left(\sum_{k=1}^{N} \sum_{(x, \xi) \in \Lambda_{k}-z_{n} \cap Q_{R}(0)}\left|\left\langle f, M_{\xi} T_{x} g_{k}\right\rangle\right|^{2}\right)^{1 / 2} \mid \\
& \leq\left(\sum_{k=1}^{N} \sum_{(x, \xi) \in \Lambda_{k}-z_{n} \cap Q_{R}(0)}\left|\left\langle f, M_{\xi+\eta(x, \xi, k)} T_{x+u(x, \xi, k)} g_{k}\right\rangle-\left\langle f, M_{\xi} T_{x} g_{k}\right\rangle\right|^{2}\right)^{1 / 2} \\
& =\left(\sum_{k=1}^{N} \sum_{(x, \xi) \in \Lambda_{k}-z_{n} \cap Q_{R}(0)}\left|\left\langle f, M_{\xi+\eta(x, \xi, k)} T_{x+u(x, \xi, k)} g_{k}-M_{\xi} T_{x} g_{k}\right\rangle\right|^{2}\right)^{1 / 2} \\
& \leq\left(\sum_{k=1}^{N} \sum_{(x, \xi) \in \Lambda_{k}-z_{n} \cap Q_{R}(0)}\|f\|_{2}^{2}\left\|M_{\xi+\eta(x, \xi, k)} T_{x+u(x, \xi, k)} g_{k}-M_{\xi} T_{x} g_{k}\right\|_{2}^{2}\right)^{1 / 2} \\
& <\left(N D\|f\|_{2}^{2} \frac{\varepsilon^{2}}{4 N D\|f\|_{2}^{2}}\right)^{1 / 2}=\frac{\varepsilon}{2} .
\end{aligned}
$$

If $D=0$, then the quantity being estimated above is zero. In any case, if we write $z_{n}=\left(v_{n}, \mu_{n}\right)$, then it follows that

$$
\begin{aligned}
& \left(\sum_{k=1}^{N} \sum_{(x, \xi) \in \Lambda_{k}^{\prime}}\left|\left\langle f, M_{\xi} T_{x} g\right\rangle\right|^{2}\right)^{1 / 2} \\
& \leq\left(\sum_{k=1}^{N} \sum_{(x, \xi) \in \Lambda_{k}^{\prime} \cap Q_{R}(0)}\left|\left\langle f, M_{\xi} T_{x} g\right\rangle\right|^{2}\right)^{1 / 2} \\
& \left.\quad+\left(\sum_{k=1}^{N} \sum_{(x, \xi) \in \Lambda_{k}^{\prime} \backslash Q_{R}(0)}\left|\left\langle f, M_{\xi} T_{x} g\right\rangle\right|^{2}\right)^{1 / 2}\left|\left\langle f, M_{\xi} T_{x} g\right\rangle\right|^{2}\right)^{1 / 2}+\frac{\varepsilon}{2}+\frac{\varepsilon}{2} \quad \text { by }(13) \text { and }(14) \\
& \leq\left(\sum_{k=1}^{N} \sum_{(x, \xi) \in \Lambda_{k}-z_{n} \cap Q_{R}(0)} \sum_{k=1}^{N} \sum_{(x, \xi) \in \Lambda_{k}}\left|\left\langle M_{\mu_{n}} T_{v_{n}} f, M_{\xi} T_{x} g\right\rangle\right|^{2}\right)^{1 / 2}+\varepsilon \\
& \leq B^{1 / 2}\left\|M_{\mu_{n}} T_{v_{n}} f\right\|_{2}+\varepsilon=B^{1 / 2}\|f\|_{2}+\varepsilon
\end{aligned}
$$

Similarly, 


$$
\begin{aligned}
& \left(\sum_{k=1}^{N} \sum_{(x, \xi) \in \Lambda_{k}^{\prime}}\left|\left\langle f, M_{\xi} T_{x} g\right\rangle\right|^{2}\right)^{1 / 2} \\
& \geq\left(\sum_{k=1}^{N} \sum_{(x, \xi) \in \Lambda_{k}^{\prime} \cap Q_{R}(0)}\left|\left\langle f, M_{\xi} T_{x} g\right\rangle\right|^{2}\right)^{1 / 2} \\
& \geq\left(\sum_{k=1}^{N} \sum_{(x, \xi) \in \Lambda_{k}-z_{n} \cap Q_{R}(0)}\left|\left\langle f, M_{\xi} T_{x} g\right\rangle\right|^{2}\right)^{1 / 2}-\frac{\varepsilon}{2} \text { by }(13) \\
& =\left(\sum_{k=1}^{N} \sum_{(x, \xi) \in \Lambda_{k}-z_{n}}\left|\left\langle f, M_{\xi} T_{x} g\right\rangle\right|^{2}-\sum_{k=1}^{N} \sum_{(x, \xi) \in \Lambda_{k}-z_{n} \backslash Q_{R}(0)}\left|\left\langle f, M_{\xi} T_{x} g\right\rangle\right|^{2}\right)^{1 / 2}-\frac{\varepsilon}{2} \\
& \geq\left(\sum_{k=1}^{N} \sum_{(x, \xi) \in \Lambda_{k}-z_{n}}\left|\left\langle f, M_{\xi} T_{x} g\right\rangle\right|^{2}\right)^{1 / 2} \\
& \left.\geq A^{1 / 2} \| \sum_{k=1}^{N} \sum_{(x, \xi) \in \Lambda_{k}-z_{n} \backslash Q_{R}(0)}\left|\left\langle f, M_{\xi} T_{x} g\right\rangle\right|^{2}\right)^{1 / 2}-\frac{\varepsilon}{2}
\end{aligned}
$$

Since $\varepsilon$ is arbitrary, we conclude that

$$
\forall f \in M^{1}\left(\mathbf{R}^{d}\right), \quad A\|f\|_{2}^{2} \leq \sum_{k=1}^{N} \sum_{(x, \xi) \in \Lambda_{k}^{\prime}}\left|\left\langle f, M_{\xi} T_{x} g\right\rangle\right|^{2} \leq B\|f\|_{2}^{2} .
$$

Since $M^{1}\left(\mathbf{R}^{d}\right)$ is dense in $L^{2}\left(\mathbf{R}^{d}\right)$, this inequality extends to all $f \in L^{2}\left(\mathbf{R}^{d}\right)$, which completes the proof.

\section{Density of Gabor Frames}

We now develop the main results on the Homogeneous Approximation Property and the density of Gabor frames.

First we define the Homogeneous Approximation Property (HAP) introduced by Ramanathan and Steger and some variations on this theme. The particular HAP introduced in [47] will here be called the Ramanathan-Steger Weak HAP. We also introduce a slightly stronger property that we call the Ramanathan-Steger Strong HAP. In [27], Gröchenig and Razafinjatovo introduced a modified version of the Ramanathan-Steger Weak HAP. The analogue of their definition for the case of Gabor systems will be called the Weak HAP. This Weak HAP was used in [11] to give a complete proof of the Density Theorem for Gabor frames. We also define a slightly stronger version that we call the Strong HAP. 
Remark 20. The terminology for Weak and Strong HAP used here is consistent with that used in [4], but differs from that used in [11]. Specifically, the definition of what was called "Strong HAP" in [11] was equivalent to the definition of the "Weak HAP" in [11], and both of those coincide with the definition of the Weak HAP used in this chapter.

\subsection{Definition of the HAP}

Definition 21. Let $g_{1}, \ldots, g_{N} \in L^{2}\left(\mathbf{R}^{d}\right) \backslash\{0\}$ and $\Lambda_{1}, \ldots, \Lambda_{N} \subset \mathbf{R}^{2 d}$ be such that

$$
\mathcal{G}=\bigcup_{k=1}^{N} \mathcal{G}\left(g_{k}, \Lambda_{k}\right)=\bigcup_{k=1}^{N}\left\{M_{\xi} T_{x} g_{k}\right\}_{(x, \xi) \in \Lambda_{k}}
$$

is a frame $L^{2}\left(\mathbf{R}^{d}\right)$. Let

$$
\tilde{\mathcal{G}}=\bigcup_{k=1}^{N}\left\{\tilde{g}_{x, \xi, k}\right\}_{(x, \xi) \in \Lambda_{k}}
$$

denote the canonical dual frame of $\mathcal{G}$ (in general, $\tilde{\mathcal{G}}$ need not itself be a Gabor frame). For each $h>0$ and $(u, \eta) \in \mathbf{R}^{2 d}$, set

$$
\begin{aligned}
& W(h, u, \eta)=\operatorname{span}\left\{\tilde{g}_{x, \xi, k}:(x, \xi) \in \Lambda_{k} \cap Q_{h}(u, \eta), k=1, \ldots, N\right\}, \\
& \tilde{W}(h, u, \eta)=\operatorname{span}\left\{M_{\xi} T_{x} g_{k}:(x, \xi) \in \Lambda_{k} \cap Q_{h}(u, \eta), k=1, \ldots, N\right\} .
\end{aligned}
$$

(a) We say that $\mathcal{G}$ possesses the Ramanathan-Steger Weak Homogeneous Approximation Property (R-S Weak HAP) if

$$
\begin{aligned}
\forall f \in L^{2}\left(\mathbf{R}^{d}\right), \quad \forall \varepsilon>0, \quad \exists R>0 \quad \text { such that } \quad \forall(u, \eta) \in \mathbf{R}^{2 d}, \\
\\
\operatorname{dist}\left(M_{\eta} T_{u} f, \tilde{W}(R, u, \eta)\right)<\varepsilon .
\end{aligned}
$$

(b) We say that $\mathcal{G}$ possesses the Weak Homogeneous Approximation Property (Weak HAP) if

$$
\begin{aligned}
\forall f \in L^{2}\left(\mathbf{R}^{d}\right), \quad \forall \varepsilon>0, \quad \exists R>0 \quad \text { such that } \quad \forall(u, \eta) \in \mathbf{R}^{2 d}, \\
\\
\operatorname{dist}\left(M_{\eta} T_{u} f, W(R, u, \eta)\right)<\varepsilon .
\end{aligned}
$$

(c) We say that $\mathcal{G}$ possesses the Ramanathan-Steger Strong Homogeneous Approximation Property (R-S Strong HAP) if

$$
\begin{gathered}
\forall f \in L^{2}\left(\mathbf{R}^{d}\right), \quad \forall \varepsilon>0, \quad \exists R>0 \quad \text { such that } \forall(u, \eta) \in \mathbf{R}^{2 d}, \\
\left\|M_{\eta} T_{u} f-\sum_{k=1}^{N} \sum_{(x, \xi) \in \Lambda_{k} \cap Q_{R}(u, \eta)}\left\langle M_{\eta} T_{u} f, \tilde{g}_{x, \xi, k}\right\rangle M_{\xi} T_{x} g_{k}\right\|_{2}<\varepsilon .
\end{gathered}
$$


(d) We say that $\mathcal{G}$ possesses the Strong Homogeneous Approximation Property (Strong HAP) if

$$
\begin{gathered}
\forall f \in L^{2}\left(\mathbf{R}^{d}\right), \quad \forall \varepsilon>0, \quad \exists R>0 \quad \text { such that } \quad \forall(u, \eta) \in \mathbf{R}^{2 d}, \\
\left\|M_{\eta} T_{u} f-\sum_{k=1}^{N} \sum_{(x, \xi) \in \Lambda_{k} \cap Q_{R}(u, \eta)}\left\langle M_{\eta} T_{u} f, M_{\xi} T_{x} g_{k}\right\rangle \tilde{g}_{x, \xi, k}\right\|_{2}<\varepsilon .
\end{gathered}
$$

Note that since the function

$$
\sum_{k=1}^{N} \sum_{(x, \xi) \in \Lambda_{k} \cap Q_{R}(u, \eta)}\left\langle M_{\eta} T_{u} f, M_{\xi} T_{x} g_{k}\right\rangle \tilde{g}_{x, \xi, k}
$$

is one element of the space $W(R, u, \eta)$, the Strong HAP implies the Weak HAP. A argument similar to [5, Thm. 5.1(e)] can be used to show that if $\mathcal{G}$ is a Riesz basis, then the Weak HAP implies the Strong HAP. Similar remarks apply to the R-S Strong and Weak HAPs.

\subsection{The HAP for Gabor Frames}

Now we establish that every Gabor frame with finitely many generators satisfies the R-S Weak HAP (in comparison, the argument in [11] shows that every Gabor frame with finitely many generators satisfies the Strong HAP).

Theorem 22. If $g_{1}, \ldots, g_{N} \in L^{2}\left(\mathbf{R}^{d}\right) \backslash\{0\}$ and $\Lambda_{1}, \ldots, \Lambda_{N} \subset \mathbf{R}^{2 d}$ are such that $\mathcal{G}=\bigcup_{k=1}^{N} \mathcal{G}\left(g_{k}, \Lambda_{k}\right)$ is a frame for $L^{2}\left(\mathbf{R}^{d}\right)$, then $\mathcal{G}$ possesses the $R-S$ Weak HAP.

Proof. By Proposition 15, we have $D^{+}\left(\Lambda_{k}\right)<\infty$ for each $k$. Therefore each $\Lambda_{k}$ is the union of finitely many uniformly separated subsequences. Hence, by passing to subsequences if necessary we may assume that each $\Lambda_{k}$ is $\delta_{k^{-}}$ uniformly separated for some $\delta_{k}>0$. Set $\delta=\min \left\{\delta_{1} / 4, \ldots, \delta_{N} / 4\right\}$. Then each $\Lambda_{k}$ is $2 \delta$-uniformly separated.

Let

$$
\tilde{\mathcal{G}}=\bigcup_{k=1}^{N}\left\{\tilde{g}_{x, \xi, k}\right\}_{(x, \xi) \in \Lambda_{k}}
$$

denote the canonical dual frame of $\mathcal{G}$, and let $A, B$ be frame bounds for $\mathcal{G}$.

Suppose that the R-S Weak HAP fails. Then there exists an $f \in L^{2}\left(\mathbf{R}^{d}\right)$ and an $\varepsilon>0$ such that for each $n \in \mathbf{N}$ we can find a point $z_{n}=\left(u_{n}, \eta_{n}\right) \in \mathbf{R}^{2 d}$ such that

$$
\operatorname{dist}\left(M_{\eta_{n}} T_{u_{n}} f, \tilde{W}\left(n, u_{n}, \eta_{n}\right)\right) \geq \varepsilon,
$$

or, equivalently,

$$
\operatorname{dist}\left(M_{\eta_{n}} T_{u_{n}} f, \operatorname{span}\left\{M_{\xi} T_{x} g_{k}:(x, \xi) \in \Lambda_{k} \cap Q_{n}\left(z_{n}\right), k=1, \ldots, N\right\}\right) \geq \varepsilon .
$$


Hence,

$$
\operatorname{dist}\left(f, \operatorname{span}\left\{M_{\xi} T_{x} g_{k}:(x, \xi) \in \Lambda_{k}-z_{n} \cap Q_{n}(0), k=1, \ldots, N\right\}\right) \geq \varepsilon .
$$

By Lemma 17, there exists a subsequence $\left\{w_{n}\right\}_{n \in \mathbf{N}}$ of $\left\{z_{n}\right\}_{n \in \mathbf{N}}$ and there exist $\Lambda_{k}^{\prime} \subset \mathbf{R}^{2 d}$ such that for each $k=1, \ldots, N$ we have

$$
\Lambda_{k}-w_{n} \stackrel{\mathrm{w}}{\longrightarrow} \Lambda_{k}^{\prime} \quad \text { as } n \rightarrow \infty .
$$

Therefore, by Lemma $19, \mathcal{G}^{\prime}=\bigcup_{k=1}^{N} \mathcal{G}\left(g_{k}, \Lambda_{k}^{\prime}\right)$ is a frame for $L^{2}\left(\mathbf{R}^{d}\right)$ with frame bounds $A, B$.

We claim now that, for any $R>0$,

$$
\operatorname{dist}\left(f, \operatorname{span}\left\{M_{\xi} T_{x} g_{k}:(x, \xi) \in \Lambda_{k}^{\prime} \cap Q_{R}(0), k=1, \ldots, N\right\}\right) \geq \frac{\varepsilon}{2} .
$$

To see this, choose any scalars $\left\{c_{k, x, \xi}\right\}_{(x, \xi) \in \Lambda_{k}^{\prime} \cap Q_{R}(0), k=1, \ldots, N}$. Let

$$
D=\sum_{k=1}^{N} \sum_{(x, \xi) \in \Lambda_{k}^{\prime} \cap Q_{R}(0)}\left|c_{k, x, \xi}\right| .
$$

We already know from equation (21) that $\|f-0\|_{2} \geq \varepsilon$, so we may assume that $D \neq 0$.

By Lemma 8 , there exists $\theta<\delta / 2$ such that

$\forall|x|,|\xi|<\theta, \forall u, \eta \in \mathbf{R}^{d}, \quad\left\|M_{\xi+\eta} T_{x+u} g_{k}-M_{\eta} T_{u} g_{k}\right\|_{2}<\frac{\varepsilon}{2 D}, \quad k=1, \ldots, N$.

As in the proof of Lemma 19, we can find $n$ large enough that each point of $\Lambda_{k}^{\prime} \cap Q_{R}(0)$ is within $\theta$ of a unique point in $\Lambda_{k}-w_{n} \cap Q_{R}(0)$, and conversely. So, we can write

$$
\begin{aligned}
\Lambda_{k}-w_{n} & \cap Q_{R}(0) \\
= & \left\{(x+u(x, \xi, k), \xi+\eta(x, \xi, k)):(x, \xi) \in \Lambda_{k}^{\prime} \cap Q_{R}(0), k=1, \ldots, N\right\},
\end{aligned}
$$

with $|x+u(x, \xi, k)|,|\xi+\eta(x, \xi, k)|<\theta$. Hence

$$
\begin{aligned}
\left\|f-\sum_{k=1}^{N} \sum_{(x, \xi) \in \Lambda_{k}^{\prime} \cap Q_{R}(0)} c_{k, x, \xi} M_{\xi} T_{x} g_{k}\right\|_{2} \\
=\| f-\sum_{k=1}^{N} \sum_{(x, \xi) \in \Lambda_{k}-w_{n} \cap Q_{R}(0)} c_{k, x, \xi} M_{\xi} T_{x} g_{k} \\
\quad+\sum_{k=1}^{N} \sum_{(x, \xi) \in \Lambda_{k}^{\prime} \cap Q_{R}(0)} c_{k, x, \xi} M_{\xi+\eta(x, \xi, k)} T_{x+u(x, \xi, k)} g_{k} \\
\quad-\sum_{k=1}^{N} \sum_{(x, \xi) \in \Lambda_{k}^{\prime} \cap Q_{R}(0)} c_{k, x, \xi} M_{\xi} T_{x} g_{k} \|_{2}
\end{aligned}
$$




$$
\begin{aligned}
& \geq\left\|f-\sum_{k=1}^{N} \sum_{(x, \xi) \in \Lambda_{k}-w_{n} \cap Q_{R}(0)} c_{k, x, \xi} M_{\xi} T_{x} g_{k}\right\|_{2} \\
& \text { - }\left\|\sum_{k=1}^{N} \sum_{(x, \xi) \in \Lambda_{k}^{\prime} \cap Q_{R}(0)} c_{k, x, \xi}\left(M_{\xi+\eta(x, \xi, k)} T_{x+u(x, \xi, k)} g_{k}-M_{\xi} T_{x} g_{k}\right)\right\|_{2} \\
& \geq \operatorname{dist}\left(f, \operatorname{span}\left\{M_{\xi} T_{x} g_{k}:(x, \xi) \in \Lambda_{k}-w_{n} \cap Q_{R}(0), k=1, \ldots, N\right\}\right) \\
& -\sum_{k=1}^{N} \sum_{(x, \xi) \in \Lambda_{k}^{\prime} \cap Q_{R}(0)}\left|c_{k, x, \xi}\right|\left\|M_{\xi+\eta(x, \xi, k)} T_{x+u(x, \xi, k)} g_{k}-M_{\xi} T_{x} g_{k}\right\|_{2} \\
& >\varepsilon-\sum_{k=1}^{N} \sum_{(x, \xi) \in \Lambda_{k}^{\prime} \cap Q_{R}(0)}\left|c_{k, x, \xi}\right| \frac{\varepsilon}{2 D}=\frac{\varepsilon}{2} .
\end{aligned}
$$

Since this is true for every choice of scalars, we conclude that equation (22) holds. But, since $R$ is arbitrary, this implies that $f \notin \overline{\operatorname{span}}(\mathcal{G})$, which contradicts the fact that $\mathcal{G}$ is complete (since it is a frame).

\subsection{The Comparison Theorem}

We saw in Theorem 22 that all Gabor frames possess the R-S Weak HAP. We will show in this section that the R-S Weak HAP implies that certain density conditions must be satisfied in comparison to any other Gabor Riesz sequence.

Theorem 23 (Comparison Theorem). Assume that

(a) $g_{1}, \ldots, g_{N} \in L^{2}\left(\mathbf{R}^{d}\right) \backslash\{0\}$ and $\Lambda_{1}, \ldots, \Lambda_{N} \subset \mathbf{R}^{2 d}$ are such that

$$
\mathcal{G}=\bigcup_{k=1}^{N} \mathcal{G}\left(g_{k}, \Lambda_{k}\right)
$$

is a frame for $L^{2}\left(\mathbf{R}^{d}\right)$, and

(b) $\phi_{1}, \ldots, \phi_{M} \in L^{2}\left(\mathbf{R}^{d}\right)$ and $\Delta_{1}, \ldots, \Delta_{M} \subset \mathbf{R}^{d}$ are such that

$$
\Phi=\bigcup_{k=1}^{M} \mathcal{G}\left(\phi_{k}, \Delta_{k}\right)
$$

is a Riesz sequence in $L^{2}\left(\mathbf{R}^{d}\right)$.

Set $\Lambda=\bigcup_{k=1}^{N} \Lambda_{k}$ and $\Delta=\bigcup_{k=1}^{M} \Delta_{k}$. Then

$$
D^{-}(\Delta) \leq D^{-}(\Lambda) \quad \text { and } \quad D^{+}(\Delta) \leq D^{+}(\Lambda) .
$$


Proof. Note that, by Theorem 22 , we have that $\mathcal{G}$ possesses the R-S Weak HAP.

We are given that $\Phi$ is a Riesz basis for its closed span within $L^{2}\left(\mathbf{R}^{d}\right)$. Let

$$
\tilde{\Phi}=\bigcup_{k=1}^{M}\left\{\tilde{\phi}_{x, \xi, k}\right\}_{(x, \xi) \in \Delta_{k}}
$$

denote the dual frame within that closed span.

Given $h>0$ and $(u, \eta) \in \mathbf{R}^{2 d}$, set

$$
\begin{aligned}
\tilde{W}(h, u, \eta) & =\operatorname{span}\left\{M_{\xi} T_{x} g_{k}:(x, \xi) \in \Lambda_{k} \cap Q_{h}(u, \eta), k=1, \ldots, N\right\}, \\
V(h, u, \eta) & =\operatorname{span}\left\{M_{\xi} T_{x} \phi_{k}:(x, \xi) \in \Delta_{k} \cap Q_{h}(u, \eta), k=1, \ldots, M\right\} .
\end{aligned}
$$

By Proposition 15 we have $D^{+}\left(\Lambda_{k}\right), D^{+}\left(\Delta_{k}\right)<\infty$ for each $k$, so these are finite-dimensional spaces.

Fix any $\varepsilon>0$. Applying the definition of the R-S Weak HAP to the functions $f=\phi_{k}$, we see that there exists an $R>0$ such that

$$
\forall(u, \eta) \in \mathbf{R}^{2 d}, \quad \operatorname{dist}\left(M_{\eta} T_{u} \phi_{k}, \tilde{W}(R, u, \eta)\right)<\frac{\varepsilon}{D}, \quad k=1, \ldots, M,
$$

where

$$
D=\sup \left\{\left\|\tilde{\phi}_{u, \eta, k}\right\|:(u, \eta) \in \Delta_{k}, k=1, \ldots, M\right\} .
$$

Fix any $h>0$ and $(u, \eta) \in \mathbf{R}^{2 d}$. Let $P_{V}$ and $P_{W}$ denote the orthogonal projections of $L^{2}\left(\mathbf{R}^{d}\right)$ onto $V=V(h, u, \eta)$ and $W=\tilde{W}(R+h, u, \eta)$, respectively. Define $T: V \rightarrow V$ by $T=P_{V} P_{W}=P_{V} P_{W} P_{V}$. Note that $T$ is self-adjoint and $V$ is finite-dimensional, so $T$ has a finite, real trace.

We will estimate the trace of $T$. First, every eigenvalue $\lambda$ of $T$ satisfies $|\lambda| \leq\|T\| \leq\left\|P_{V}\right\|\left\|P_{W}\right\|=1$. Since the trace is the sum of the nonzero eigenvalues, this provides us with an upper bound for the trace:

$$
\operatorname{trace}(T) \leq \operatorname{rank}(T) \leq \operatorname{dim}(W)=\#\left(\Lambda \cap Q_{R+h}(u, \eta)\right) .
$$

For a lower estimate, note that

$$
\left\{M_{\xi} T_{x} \phi_{k}:(x, \xi) \in \Delta_{k} \cap Q_{h}(u, \eta), k=1, \ldots, M\right\}
$$

is a basis for the finite-dimensional space $V$ (since $\Phi$ is a Riesz sequence and hence is linearly independent). The dual basis in $V$ is the biorthogonal system in $V$, which is

$$
\left\{P_{V} \tilde{\phi}_{x, \xi, k}:(x, \xi) \in \Delta_{k} \cap Q_{h}(u, \eta), k=1, \ldots, M\right\} .
$$

Therefore,

$$
\begin{aligned}
\operatorname{trace}(T) & =\sum_{k=1}^{M} \sum_{(x, \xi) \in \Delta_{k} \cap Q_{h}(u, \eta)}\left\langle T\left(M_{\xi} T_{x} \phi_{k}\right), P_{V} \tilde{\phi}_{x, \xi, k}\right\rangle \\
& =\sum_{k=1}^{M} \sum_{(x, \xi) \in \Delta_{k} \cap Q_{h}(u, \eta)}\left\langle P_{V} P_{W}\left(M_{\xi} T_{x} \phi_{k}\right), P_{V} \tilde{\phi}_{x, \xi, k}\right\rangle
\end{aligned}
$$




$$
\begin{aligned}
& =\sum_{k=1}^{M} \sum_{(x, \xi) \in \Delta_{k} \cap Q_{h}(u, \eta)}\left\langle P_{W}\left(M_{\xi} T_{x} \phi_{k}\right), P_{V} \tilde{\phi}_{x, \xi, k}\right\rangle \\
& =\sum_{k=1}^{M} \sum_{(x, \xi) \in \Delta_{k} \cap Q_{h}(u, \eta)}\left(\left\langle M_{\xi} T_{x} \phi_{k}, P_{V} \tilde{\phi}_{x, \xi, k}\right\rangle\right. \\
& \left.+\left\langle\left(P_{W}-I\right)\left(M_{\xi} T_{x} \phi_{k}\right), P_{V} \tilde{\phi}_{x, \xi, k}\right\rangle\right) .
\end{aligned}
$$

By the biorthogonality of $\Phi$ and $\tilde{\Phi}$,

$$
\left\langle M_{\xi} T_{x} \phi_{k}, P_{V} \tilde{\phi}_{x, \xi, k}\right\rangle=1 .
$$

Additionally, if $(x, \xi) \in Q_{h}(u, \eta)$ then we have $Q_{R}(x, \xi) \subset Q_{R+h}(u, \eta)$, so $W(R, x, \xi) \subset W(R+h, u, \eta)$ and therefore

$$
\begin{aligned}
\left|\left\langle\left(P_{W}-I\right)\left(M_{\xi} T_{x} \phi_{k}\right), P_{V} \tilde{\phi}_{x, \xi, k}\right\rangle\right| & \leq\left\|\left(P_{W}-I\right)\left(M_{\xi} T_{x} \phi_{k}\right)\right\|_{2}\left\|P_{V} \tilde{\phi}_{x, \xi, k}\right\| \\
& \leq \operatorname{dist}\left(M_{\xi} T_{x} \phi_{k}, W(R+h, u, \eta)\right)\left\|\tilde{\phi}_{x, \xi, k}\right\|_{2} \\
& \leq \operatorname{dist}\left(M_{\xi} T_{x} \phi_{k}, W(R, x, \xi)\right) D \\
& \leq \frac{\varepsilon}{D} D=\varepsilon .
\end{aligned}
$$

Combining (25)-(27) yields the lower bound

$$
\operatorname{trace}(T) \geq \sum_{k=1}^{M} \sum_{(x, \xi) \in \Delta_{k} \cap Q_{h}(x, \xi)}(1-\varepsilon)=(1-\varepsilon) \#\left(\Delta \cap Q_{h}(u, \eta)\right) .
$$

Finally, combining the upper estimate (24) with the lower estimate (28), we see that

$$
\forall(u, \eta) \in \mathbf{R}^{2 d}, \quad \forall h>0, \quad(1-\varepsilon) \#\left(\Delta \cap Q_{h}(u, \eta)\right) \leq \#\left(\Lambda \cap Q_{R+h}(u, \eta)\right),
$$

and so

$$
\begin{aligned}
D^{-}(\Delta) & =\liminf _{h \rightarrow \infty} \inf _{(u, \eta) \in \mathbf{R}^{2 d}} \frac{\#\left(\Delta \cap Q_{h}(u, \eta)\right)}{h^{2 d}} \\
& \leq \frac{1}{1-\varepsilon} \liminf _{h \rightarrow \infty} \inf _{(u, \eta) \in \mathbf{R}^{2 d}} \frac{\#\left(\Lambda \cap Q_{R+h}(u, \eta)\right)}{(R+h)^{2 d}} \frac{(R+h)^{2 d}}{h^{2 d}} \\
& =\frac{1}{1-\varepsilon} D^{-}(\Lambda) .
\end{aligned}
$$

Since $\varepsilon$ is arbitrary, we conclude that $D^{-}(\Delta) \leq D^{-}(\Lambda)$, and a similar calculation shows that $D^{+}(\Delta) \leq D^{+}(\Lambda)$. 


\subsection{The Density Theorem}

Combining our previous results on the HAP and the Comparison Theorem, yields a proof of an extended version of the Density Theorem.

Theorem 24 (Density Theorem). Let $g_{1}, \ldots, g_{N} \in L^{2}\left(\mathbf{R}^{d}\right) \backslash\{0\}$ and $\Lambda_{1}, \ldots, \Lambda_{N} \subset \mathbf{R}^{2 d}$ be given. Let

$$
\mathcal{G}=\bigcup_{k=1}^{N} \mathcal{G}\left(g_{k}, \Lambda_{k}\right) \quad \text { and } \quad \Lambda=\bigcup_{k=1}^{N} \Lambda_{k} .
$$

Then the following statements hold.

(a) If $\mathcal{G}$ is a frame for $L^{2}\left(\mathbf{R}^{d}\right)$, then $1 \leq D^{-}(\Lambda) \leq D^{+}(\Lambda)<\infty$.

(b) If $\mathcal{G}$ is a Riesz sequence in $L^{2}\left(\mathbf{R}^{d}\right)$, then $0 \leq D^{-}(\Lambda) \leq D^{+}(\Lambda) \leq 1$.

(c) If $\mathcal{G}$ is a Riesz basis for $L^{2}\left(\mathbf{R}^{d}\right)$, then $D^{-}(\Lambda)=D^{+}(\Lambda)=1$.

Proof. Define $\phi=\chi_{Q_{1}(0)}$ and $\Delta=\mathbf{Z}^{d}$. Note that $\Phi=\mathcal{G}(\phi, \Delta)$ is an orthonormal basis for $L^{2}\left(\mathbf{R}^{d}\right)$, and hence is both a frame and a Riesz basis. Also, $D^{ \pm}(\Delta)=1$.

(a) Assume that $\mathcal{G}$ is a frame for $L^{2}\left(\mathbf{R}^{d}\right)$. Then $D^{+}\left(\Lambda_{k}\right)<\infty$ for each $k$ by Lemma 15 , so $D^{+}(\Lambda)<\infty$ as well. Further, applying Theorem 23 to $\mathcal{G}$ and $\Phi$ implies that $D^{-}(\Lambda) \geq D^{-}(\Delta)=1$.

(b) Suppose that $\mathcal{G}$ is a Riesz sequence in $L^{2}\left(\mathbf{R}^{d}\right)$. Then applying Theorem 23 to $\Phi$ and $\mathcal{G}$ yields $1=D^{-}(\Delta) \geq D^{-}(\Lambda)$.

(c) If $\mathcal{G}$ is a Riesz basis, then it is both a frame and a Riesz sequence, so parts (a) and (b) together imply that $D^{-}(\Lambda)=D^{+}(\Lambda)=1$.

\section{Acknowledgments}

During the years 2002-2006, I had the honor of being part of an NSF-sponsored Focused Research Group (FRG) along with seven other wonderful people, including Larry. This was my first nontrivial interaction with Larry, and I quickly grew to greatly respect him both as a mathematician and a friend. Of all the many good things that came out of that FRG, I value the fact that it brought me into contact with Larry among the highest.

Larry's incompleteness result in [2] was one of the explicit beginnings of the Density Theorem for Gabor frames, and it was the inspiration for the talk that I gave at the conference held in honor of Larry at the University of Colorado in May 2006. The history of the Density Theorem given in this chapter is adapted from that talk.

The accounting of the Ramanathan-Steger proof given in the body of this chapter is inspired by discussions by the author with Gerard Ascensi in Spring 2005. We also thank Gitta Kutyniok for valuable discussions, and we are indebted to Jay Ramanathan for many collaborations and insights.

The support of NSF Grant DMS-0139261 is gratefully acknowledged. 


\section{References}

1. H. Bacry, A. Grossmann, and J. Zak, Proof of completeness of lattice states in the kq representation, Phys. Rev. B, 12 (1975), 1118-1120.

2. L. Baggett, Processing a radar signal and representations of the discrete Heisenberg group, Colloq. Math., 60/61 (1990), 195-203.

3. R. Balan, P. G. Casazza, C. Heil, and Z. Landau, Excesses of Gabor frames, Appl. Comput. Harmon. Anal., 14 (2003), 87-106.

4. R. Balan, P. G. Casazza, C. Heil, and Z. Landau, Density, overcompleteness, and localization of frames, I. Theory, J. Fourier Anal. Appl., 12 (2006), 105-143.

5. R. Balan, P. G. Casazza, C. Heil, and Z. Landau, Density, overcompleteness, and localization of frames, II. Gabor systems, J. Fourier Anal. Appl., 12 (2006), 307-344.

6. V. Bargmann, P. Butera, L. Girardello, and J. R. Klauder, On the completeness of coherent states, Rep. Math. Phys., 2 (1971), 221-228.

7. B. Bekka, Square integrable representations, von Neumann algebras and an application to Gabor analysis, J. Fourier Anal. Appl., 10 (2004), 325-349.

8. J. J. Benedetto, C. Heil, and D. F. Walnut, Differentiation and the Balian-Low theorem, J. Fourier Anal. Appl., 1 (1995), 355-402.

9. M. Bownik and Z. Rzeszotnik, The spectral function of shift-invariant spaces, Michigan Math. J., 51 (2003), 387-414.

10. O. Christensen, An Introduction to Frames and Riesz Bases, Birkhäuser, Boston, 2003.

11. O. Christensen, B. Deng, and C. Heil, Density of Gabor frames, Appl. Comput. Harmon. Anal., 7 (1999), 292-304.

12. O. Christensen, S. Favier, and Z. Felipe, Irregular wavelet frames and Gabor frames, Approx. Theory Appl. (N.S.), 17 (2001), 90-101.

13. I. Daubechies, The wavelet transform, time-frequency localization and signal analysis, IEEE Trans. Inform. Theory, 39 (1990), 961-1005.

14. I. Daubechies, Ten Lectures on Wavelets, SIAM, Philadelphia, 1992.

15. I. Daubechies and A. Grossmann, Frames in the Bargmann space of entire functions, Comm. Pure Appl. Math., 41 (1988), 151-164.

16. I. Daubechies, A. Grossmann, and Y. Meyer, Painless nonorthogonal expansions, J. Math. Phys., 27 (1986), 1271-1283.

17. I. Daubechies, H. Landau, and Z. Landau, Gabor time-frequency lattices and the Wexler-Raz identity, J. Fourier Anal. Appl., 1 (1995), 437-478.

18. B. Deng and C. Heil, Density of Gabor Schauder bases, in: "Wavelet Applications in Signal and Image Processing VIII" (San Diego, CA, 2000), A. Aldroubi at al., eds., Proc. SPIE Vol. 4119, SPIE, Bellingham, WA, 2000, 153-164.

19. R. J. Duffin and A. C. Schaeffer, A class of nonharmonic Fourier series, Trans. Amer. Math. Soc., 72 (1952), 341-366.

20. H. G. Feichtinger and W. Kozek, Quantization of TF lattice-invariant operators on elementary LCA groups, in: "Gabor Analysis and Algorithms: Theory and Applications," H. G. Feichtinger and T. Strohmer, eds., Birkhäuser, Boston, 1998, 233-266.

21. G. B. Folland, The abstruse meets the applicable: Some aspects of timefrequency analysis, Proc. Indian Acad. Sci. Math. Sci., 116 (2006), 121-136.

22. J.-P. Gabardo and D. Han, Frame representations for group-like unitary operator systems, J. Operator Theory, 49 (2003), 223-244. 
23. D. Gabor, Theory of communications, J. Inst. Elec. Eng. (London), 93 (1946), 429-457.

24. K. Gröchenig, Irregular sampling of wavelet and short-time Fourier transforms, Constr. Approx., 9 (1993), 283-297.

25. K. Gröchenig, Foundations of Time-Frequency Analysis, Birkhäuser, Boston, 2001.

26. K. Gröchenig, Localization of frames, Banach frames, and the invertibility of the frame operator, J. Fourier Anal. Appl., 10 (2004), 105-132.

27. K. Gröchenig and H. Razafinjatovo, On Landau's necessary density conditions for sampling and interpolation of band-limited functions, J. London Math. Soc. (2), 54, 557-565 (1996).

28. D. Han and Y. Wang, Lattice tiling and the Weyl-Heisenberg frames, Geom. Funct. Anal., 11 (2001), 742-758.

29. C. Heil, An introduction to weighted Wiener amalgams, in: "Wavelets and their Applications" (Chennai, January 2002), M. Krishna, R. Radha and S. Thangavelu, eds., Allied Publishers, New Delhi, 2003, 183-216.

30. C. Heil, History and evolution of the Density Theorem for Gabor frames, preprint (2006).

31. C. Heil and G. Kutyniok, Density of frames and Schauder bases of windowed exponentials, preprint (2006).

32. C. Heil and G. Kutyniok, The Homogeneous Approximation Property for wavelet frames, preprint (2006).

33. C. E. Heil and D. F. Walnut, Continuous and discrete wavelet transforms, SIAM Review, 31 (1989), 628-666.

34. A. J. E. M. Janssen, Gabor representation of generalized functions, J. Math. Anal. Appl., 83 (1981), 377-394.

35. A. J. E. M. Janssen, Signal analytic proofs of two basic results on lattice expansions, Appl. Comput. Harmon. Anal., 1 (1994), 350-354.

36. A. J. E. M. Janssen, A density theorem for time-continuous filter banks, in: "Signal and Image Representation in Combined Spaces," Y. Y. Zeevi and R. R. Coifman, eds., Wavelet Anal. Appl., Vol. 7, Academic Press, San Diego, CA, 1998, $513-523$.

37. G. Kutyniok, Beurling density and shift-invariant weighted irregular Gabor systems, Sampl. Theory Signal Image Process., 5 (2006), 163-181.

38. H. Landau, Sampling, data transmission, and the Nyquist rate, Proc. IEEE, 55, 1701-1706 (1967).

39. H. Landau, Necessary density conditions for sampling and interpolation of certain entire functions, Acta Math., 117, 37-52 (1967).

40. H. Landau, On the density of phase-space expansions, IEEE Trans. Inform. Theory, 39 (1993), 1152-1156.

41. Y. Liu and Y. Wang, The uniformity of non-uniform Gabor bases, Adv. Comput. Math., 18 (2003), 345-355.

42. Yu. I. Lyubarskii, Frames in the Bargmann space of entire functions, in: "Entire and subharmonic functions," Amer. Math. Soc., Providence, RI, 1992, 167-180.

43. J. von Neumann, "Mathematische Grundlagen der Quantenmechanik," Springer, Berlin, 1932. English translation: "Mathematical foundations of quantum mechanics," Princeton University Press, Princeton, NJ, 1955.

44. A. Olevskii, Completeness in $L^{2}(\mathbf{R})$ of almost integer translates, C. R. Acad. Sci. Paris, 324 (1997), 98-991. 
45. A. Olevskii and A. Ulanovskii, Almost integer translates. Do nice generators exist?, J. Fourier Anal. Appl., 10 (2004), 93-104.

46. A. M. Perelomov, On the completeness of a system of coherent states (English translation), Theoret. Math. Phys., 6 (1971), 156-164.

47. J. Ramanathan and T. Steger, Incompleteness of sparse coherent states, Appl. Comput. Harmon. Anal., 2, 148-153 (1995).

48. M. Rieffel, Von Neumann algebras associated with pairs of lattices in Lie groups, Math. Ann., 257 (1981), 403-418.

49. E. Romero, A complete Gabor system of zero Beurling density, Sampl. Theory Signal Image Process., 3 (2004), 299-304.

50. K. Seip, Density theorems for sampling and interpolation in the Bargmann-Fock space I, J. Reine Angew. Math., 429 (1992), 91-106.

51. K. Seip and R. Wallstén, Sampling and interpolation in the Bargmann-Fock space II, J. Reine Angew. Math., 429 (1992), 107-113.

52. W. Sun and X. Zhou, Irregular wavelet/Gabor frames, Appl. Comput. Harmon. Anal., 13 (2002), 63-76.

53. W. Sun and X. Zhou, Irregular Gabor frames and their stability, Proc. Amer. Math. Soc., 131 (2003), 2883-2893.

54. R. Young, An Introduction to Nonharmonic Fourier Series, Revised First Edition, Academic Press, San Diego, 2001. 